\title{
Nonlinear water waves at a submerged obstacle or bottom topography
}

\author{
John Grue \\ Division of Mechanics, Department of Mathematics \\ University of Oslo, Norway
}

\begin{abstract}
Nonlinear diffraction of low amplitude deep water waves due to a shallowly submerged cylindrical body or an elongated bottom topography being close to the free surface is studied experimentally in a wave channel and theoretically. The wave crests and the axis of the cylinder or the the bottom topography are parallel. The incoming waves, with wave length $\lambda$, undergo strong nonlinear deformations at the shallowly submerged obstacle when the wave amplitude is not small compared to the local water depth above the obstacle. These deformations introduce an infinite number of super harmonic oscillations to the flow, generating free super harmonic waves propagating away from the from the obstacle superposed upon the reflected and transmitted waves with wave length $\lambda$. The wave lengths of the super harmonic waves are $\lambda / 4$, $\lambda / 9, \lambda / 16, \ldots$, due to the quadratic in wave frequency dispersion relation for deep water waves. Their amplitudes, growing with increasing incoming wave amplitude up to saturation values, are found to be prominent at the obstacle's lee side, while they are vanishingly small at the weather side. The second and third harmonic wave amplitudes are, surprisingly, in several examples found be of the same order of magnitude as the incoming wave amplitude. Up to $25 \%$ of the incoming energy flux may be transferred to the shorter waves. The theoretical model accounts for nonlinearity by the Boussinesq equations in the shallow region above the obstacle, with matching to transient linearized potential theory in the deep water at both sides. The theory explains both qualitatively and quantitatively the trends observed in the experiments up to breaking.
\end{abstract}

\section{Introduction}

Low amplitude ocean waves propagating over shallow reefs, sunken rocks or under water ridges may, in addition to being diffracted, be broken up into shorter super harmonic free waves due to nonlinear free surface effects. This is true for swells propagating towards the Norwegian west coast where the bottom topography many places have several shallow under water ridges rising from deep water. The generation of the super harmonic waves changes the swell spectrum because a significant part of the incoming wave energy may be transferred to higher frequencies. This phenomenon is observed if one is sailing in a small boat seaward on a day when only swells are propagating towards the shore. Close to land the boat is responding to both longer and shorter waves. By sailing seaward, across the under water ridges, the shorter waves get weaker and weaker, and in the open sea only the pure swells are observed.

The generation of free super harmonic waves also occurs at a shallowly submerged marine structure, introducing super harmonic oscillatory forces and contributions to the 
mean horizontal drift force acting upon the structure. The mean horizontal drift force due to the incoming and scattered waves, usually directed along the incoming wave direction, may be reversed when the super harmonic waves generated at the body's lee side are large.

In the present contribution we study this phenomenon by simplified examples experimentally in a wave channel and theoretically. Incoming waves with low amplitude propagating in a fluid layer with great water depth are diffracted by a submerged body or a bottom topography which is close to the free surface. The body is a horizontal circular cylinder and the bottom topography is rectangular. Both geometries have axes parallel to the crests of the incoming waves. The presence of the body or the bottom topography introduces locally a shallow water depth which leads to significant deformations of the incoming waves as they propagate from the deep fluid layer into the shallow region above the obstacle and to the deep layer again. The deformations of the waves at the obstacle are linear if the incoming wave amplitude, $a$, is small compared to the local shallow water depth. Nonlinear effects become, however, prominent at the obstacle when the ratio between $a$ and the local water depth is not small. The initially symmetric wave profile become then asymmetric and skewed, and the waves may for larger values of $a$ be spilling or plunging. The nonlinear deformations introduce, in addition to the oscillations with the same frequency as the incoming wave frequency, $\omega$, a hierarchy of super harmonic oscillations to the flow with frequencies $2 \omega, 3 \omega, 4 \omega \ldots$, which then generate trains of free waves propagating away from the obstacle. The wave length of the incoming waves, denoted by $\lambda$, is connected to the wave frequency $\omega$, through the dispersion relation

$$
\lambda=2 \pi g / \omega^{2}
$$

assuming that the water depth is greater than $\lambda$. By replacing $\omega$ by $n \omega, n=2,3,4, \ldots$, in (1) we obtain the wave lengths of the free super harmonic waves by $\lambda / 4, \lambda / 9, \lambda / 16, \ldots$, which are superposed upon the reflected and transmitted waves with wave length $\lambda$.

The aim of the paper is, by experiments and numerical simulations, to quantify the amplitudes of the super harmonic far-field waves, and to describe their generation in detail. We find that the super harmonic waves are remarkably pronounced at the obstacle's lee side. For increasing incoming wave amplitude the higher harmonics wave amplitudes are growing up to a saturation value is reached. As we shall see, the second and the third harmonic free waves may, surprisingly, attain amplitudes which are as large as the first harmonic transmitted wave amplitude. More specifically, we find that the free second and third harmonic wave amplitudes may be up to $60 \%$ of the incoming wave amplitude. This means that a significant amount of the incoming wave energy flux, up to $25 \%$ in the present examples, is transferred to the wave components being much shorter. The amplitudes of the higher harmonic waves on the weather side of the obstacle are, on the other hand, always very small, even if there is a large first harmonic reflected wave, or the incoming waves are breaking in front of the submerged obstacle. Thus, the deformations of the incoming waves introduce, practically speaking, changes in the flow only on the lee side of the obstacle.

This problem was studied experimentally and theoretically by Williams (1964) for a horizontal plate situated very close to the free surface. His wave measurements are basically obtained locally at the plate. He concludes that wave components up to the third harmonic are present locally at the body and in the far-field. His results for the far-field is, however, sparce and are complicated to compare with. We find here, for moderate incoming wave amplitude, qualitatively the same results as Williams, that oscillations up to the third harmonic are introduced to the flow. For larger values of $a$, we find, however, that very 
strong nonlinear effects take place locally at the obstacle, which means that the fourth and higher harmonic oscillations also are present.

Measurements of second harmonic free waves generated at a slightly submerged cylinder are also presented by Longuett-Higgins (1977, figure 5) in connection to measurements of negative horizontal drift forces. He finds that the second harmonic wave amplitude may be as large as approximately $45 \%$ of the incoming wave amplitude, and furthermore that it reaches a saturation value, which magnitude is not, however, given his example. Another experimental work, concerning the oscillatory first-, second- and third harmonic diffraction forces upon a submerged circular cylinder is by Chaplin (1984). He also reports measurements of the reflection power due to the circular cylinder, with the conclusion that the first- and higher harmonic reflected waves always are very small, even if wave breaking occurs at the body. We obtain here the same conclusions as Chaplin regarding the reflection power. Chaplin also mention that second harmonic free waves, with prominent amplitude, are generated at the cylinder's lee side without, however, quantifying it.

The second harmonic free waves as well as the second harmonic oscillatory forces may be computed by second order in the incoming wave amplitude potential theory (Lee 1968, Vada 1987, McIver and McIver 1990, Friis, Grue and Palm 1991) and for larger values of $a$ by nonlinear simulations, which for the submerged circular cylinder was obtained recently by Cointe (1989). We compare the measurements of the second harmonic free wave amplitude with results by the second order theory, with excellent agreement for small values of the incoming wave amplitude. Cointe's nonlinear simulations for the circle, originally compared with preliminary experiments by Grue and Granlund (1988), show striking agreement with the experiments, even for larger values of $a$ when saturation is reached.

In order to investigate the main generation mechanism of the super harmonic waves in more detail we also develop a simplified nonlinear wave diffraction model for the rectangular bottom topography case. The model accounts for nonlinearity by the Boussinesq equations in the shallow region above the bottom topography, with matching at the ends to transient, linearized potential flow, applied in the deep water. The matching procedure between the linear and nonlinear flow regimes is very effective, and the model shows good agreement with the experiments both locally at the bottom topography and in the far-field, empasizing that both nonlinearity and dispersion are important locally at the obstacle, while the linear, dispersive effects are the dominating far away.

The experimental set up and procedure for the measurements are outlined in $\S 2$. We describe in $\$ 3$ the experimental results for the super harmonic wave amplitudes in the farfield. The nonlinear diffraction theory for the rectangular bottom topography is outlined in $\S 4$ with numerical implementation and results of simulations given in $\S 5$.

\section{Experimental set up and procedure}

The experiments are carried out in a wave channel at Department of Mathematics at the University of Oslo. The channel is $14.2 \mathrm{~m}$ long, $0.47 \mathrm{~m}$ broad and is filled with water at a depth which is varied from $0.44 \mathrm{~m}$ to $0.46 \mathrm{~m}$. At one end the channel is equipped with a wave maker, a vertical rigid plate, driven by a hydraulic servo-controlled cylinder, which can perform oscillations under program control. At the other end of the channel there is a $1.5 \mathrm{~m}$ long absorbing beach, which reflects less than $10 \%$ of the incoming wave amplitude. The wave generation is very accurate and repeatable. The generator is operated such that the incoming waves are pure Stokes waves without second and higher harmonic parasittic 
free waves superposed.

The frequencies of the incoming waves are in the examples chosen as either $\omega=2 \pi \times$ $0.95 \mathrm{~Hz}, \omega=2 \pi \times 1.05 \mathrm{~Hz}$ or $\omega=2 \pi \times 1.22 \mathrm{~Hz}$, with corresponding wave lengths being $\lambda=1.62 \mathrm{~m}, \lambda=1.37 \mathrm{~m}$ or $\lambda=1.04 \mathrm{~m}$, respectively. The incoming waves are practically speaking deep water waves, since the wave lengths corresponding to the wave frequencies above for infinte water depth are respectively $\lambda=1.73 \mathrm{~m}, \lambda=1.42 \mathrm{~m}$ or $\lambda=1.05 \mathrm{~m}$. The incoming wave amplitude is varied between $2 \mathrm{~mm}$ and $28 \mathrm{~mm}$, which means that the wave slope is smaller than 0.17 .

The reflected and transmitted waves are measured for two different circular cylinders and a rectangular bottom topography, all geometries spanning the whole width of the channel. The smaller circle has radius $R=100 \mathrm{~mm}$, while the radius of the larger one is $R=190 \mathrm{~mm}$. The rectangular bottom topography has a cross section being $500 \mathrm{~mm}$ long and $410 \mathrm{~mm}$ high. The uppermost corners of the rectangle are well rounded in order to reduce flow separation which may originate there. The geometries are placed with the lee side's furthest extention a distance of $5.7 \mathrm{~m}$ from the average position of the wave maker, and with their tops positioned horizontally within a deviation less than $0.2 \mathrm{~mm}$ across the width of the channel in order to minimize cross variations in the flow which can occur at the lee side. The distance $h$ between the uppermost point of the geometries and the mean free surface is varied between $25 \mathrm{~mm}$ and $100 \mathrm{~mm}$.

The surface elevation is recorded by four wave gauges with a resolution of approximately $0.1 \mathrm{~mm}$. The gauges are static calibrated. The accuracy of the analog to digital recording of the surface elevation is tested by mounting the gauges to a motorized eccentric which forced the gauges to perform a circular path of a given radius with constant angular velocity in calm fluid. Repeated tests with this arrangement revealed that the recording of the surface elevation has a relative accuracy better than $5 \%$.

The four gauges are arranged couplewise symmetric with respect to and $12 \mathrm{~cm}$ off the centerplane of the channel, and with one couple a distance behind the other. This distance is varied between $10 \mathrm{~cm}$ and $30 \mathrm{~cm}$. The arrangement enables recording of the reflected waves by the geometry, the reflected waves by the beach, the higher harmonic free waves and the forced components of the Stokes waves. Also transverse variations, which are observed at the geometry's lee side when wave breaking occurs, are recorded by this arrangement.

First a set of runs was made with the gauges at the weather side, to measure the incoming waves and the reflection power of the obstacle. Next the runs were repeated with the gauges at the obstacle's lee side. The gauges were at the weather side and the lee side placed at different distances from the obstacle. Each experiment was running 2 minutes before the recording was started. The transients were then small. Then recording went on for 1 minute.

Wave breaking may occur at the geometry when the incoming wave amplitude is large compared to the local water depth. We here denote, as seems to be standard, the breaking wave as a plunger when air bubbles clearly are observed in the fluid. By spilling we mean a breaking process which does not give rise to air bubbles in the fluid. When wave breaking occurs at the geometry, we observe that variations along the wave crests are introduced at the lee side. The measurements reveal, however, that the variations along the crest occur only for the super harmonic waves and not for the first harmonic component. The latter result is as expected since the cross variations introduced by the breaking process contain a minor part of the incoming wave energy, introducing a correspondingly small change in the first harmonic transmitted wave, which contain the dominant part of the transmitted wave energy. The higher harmonic wave components originate, however, from the steep 
wave at the obstacle, and small cross variations there are then continued down-wave of the obstacle. In spite of the presence of the cross-variations in the wave field, the time records of the surface elevation at each geometric location show a steady pattern. This suggests that the cross variations simply are due to that the waves are propagating down the channel with crests not exactly orthogonal to the channel walls. By averaging across the channel we obtain results for the higher harmonic wave amplitudes which are almost independent of the distance from the geometry. Cross variations in a wave channel may also be due to sloshing modes or cross waves, see for example Kit, Shemer and Miloh (1987). These effects are very small in the present examples since the wave frequencies are chosen different from the cut-off frequencies and the wave amplitudes are small.

\subsection{Wave kinematics}

Let us introduce the positive $x$-axis in the mean free surface along the channel length directed towards the beach. The surface elevation $\eta_{I}$ of the incoming waves with amplitude $a$, wave number $K$ and wave frequency $\omega$, generated by the wave maker, reads

$$
\eta_{I}(x, t)=a \cos (K x-\omega t+\delta)+a_{l}^{(2)} \cos 2(K x-\omega t+\delta)+\ldots
$$

where $t$ denotes time and $\delta$ is a phase angle. $a_{l}^{(2)}$ denotes the amplitude of the forced second harmonic wave component connected to the incoming wave. For given frequency $\omega$ and water depth $H$ the wave number $K$ is obtained by the dispersion relation

$$
\omega^{2}=g K \tanh K H
$$

In the examples considered, $\tanh K H \simeq 1$, which means that $K$ deviates very little from the value of the deep water wave number given by $\omega^{2} / g$. The theoretical value of $a_{l}^{(2)}$ is thus

$$
a_{l}^{(2)} \simeq \frac{1}{2} a^{2} K
$$

which agree with the experimental observations. We assume that the reflected waves from the obstacle read

$$
\begin{aligned}
\eta_{-}(x, t)=a_{-}^{(1)} \cos \left(K x+\omega t+\delta_{-}^{(1)}\right) & +\sum_{n>1} a_{l-}^{(n)} \cos n\left(K x+\omega t+\delta_{-}^{(1)}\right) \\
& +\sum_{n>1} a_{-}^{(n)} \cos \left(K_{n} x+n \omega t+\delta_{-}^{(n)}\right)
\end{aligned}
$$

where $a_{-}^{(n)}, n=1,2, \ldots$, denote the nth harmonic reflected free wave amplitudes, $a_{l-}^{(n)}, n=$ $2,3, \ldots$, denote the amplitude of the forced nth harmonic wave components connected to $a_{-}^{(1)} \cos \left(K x+\omega t+\delta_{-}^{(1)}\right)$ and $\delta_{-}^{(n)}, n=1,2, \ldots$, are phase angles. The wave numbers $K_{n}, n=$ $2,3, \ldots$, are given by

$$
(n \omega)^{2}=g K_{n} \tanh K_{n} H, \quad n=2,3, \ldots
$$

With $\tanh K_{n} H$ very close to unity, $K_{n}$ is given by

$$
K_{n}=\frac{n^{2} \omega^{2}}{g}, \quad n=2,3, \ldots
$$


At the lee side of the cylinder we assume that the waves are given by

$$
\begin{aligned}
\eta_{+}(x, t)=a_{+}^{(1)} \cos \left(K x-\omega t+\delta_{+}^{(1)}\right) & +\sum_{n>1} a_{l+}^{(n)} \cos n\left(K x-\omega t+\delta_{+}^{(1)}\right) \\
& +\sum_{n>1} a_{+}^{(n)} \cos \left(K_{n} x-n \omega t+\delta_{+}^{(n)}\right)
\end{aligned}
$$

where $a_{+}^{(n)}, 1,2, \ldots$, denote the nth harmonic transmitted free wave amplitudes, $a_{l_{+}}^{(n)}, n=$ $2,3, \ldots$, denote the amplitudes of the forced $n$th harmonic wave components connected to the first harmonic transmitted wave and $\delta_{+}^{(n)}, n=1,2, \ldots$, are phase angles.

The free wave amplitudes, as well as the forced wave amplitudes, are obtained from the time records of the surface elevation $\eta(x, t)$. Introducing the Fourier transform by

$$
\hat{\eta}^{(n)}(x)=\frac{\omega}{2 \pi} \int_{0}^{\frac{2 \pi}{\omega}} \eta(x, t) \exp (-i n \omega t) d t, \quad n=1,2, \ldots
$$

we may obtain the incoming wave amplitude, $a$, and the reflected wave amplitude by the geometry, $a_{-}^{(1)}$, by measuring $\hat{\eta}^{(1)}$ at two positions $x_{1}$ and $x_{1}+\Delta x$ at the weather side. We find

$$
\begin{aligned}
& a=\frac{1}{|\sin (K \Delta x)|}\left|\hat{\eta}^{(1)}\left(x_{1}\right)-\hat{\eta}^{(1)}\left(x_{1}+\Delta x\right) \exp (-i K \Delta x)\right| \\
& a_{-}^{(1)}=\frac{1}{|\sin (K \Delta x)|}\left|\hat{\eta}^{(1)}\left(x_{1}\right)-\hat{\eta}^{(1)}\left(x_{1}+\Delta x\right) \exp (i K \Delta x)\right|
\end{aligned}
$$

At the lee side we obtain corresponding results for the first harmonic transmitted wave and the reflection power due to the beach. We always find that the reflection due to the beach is less than $10 \%$. The reflection by the beach is even smaller for the shorter higher harmonic wave components, which gives that the higher harmonic wave amplitudes at the lee side are obtained by

$$
\begin{array}{lr}
a_{+}^{(n)}=\frac{1}{\left|\sin \left(\frac{1}{2}\left(K_{n}-n K\right) \Delta x\right)\right|}\left|\hat{\eta}^{(n)}\left(x_{1}\right)-\hat{\eta}^{(n)}\left(x_{1}+\Delta x\right) \exp (i n K \Delta x)\right|, & n=2,3,4, \ldots \\
\left.a_{l+}^{(n)}=\frac{1}{\left|\sin \left(\frac{1}{2}\left(K_{n}-n K\right) \Delta x\right)\right|} \mid \hat{\eta}^{(n)}\left(x_{1}\right)-\hat{\eta}^{(n)}\left(x_{1}+\Delta x\right) \exp i K_{n} \Delta x\right) \mid, & n=2,3,4, \ldots
\end{array}
$$

The distance $\Delta x$ between the gauges is varied between $10 \mathrm{~cm}$ and $30 \mathrm{~cm}$. The position $x_{1}$ is at the weather side varied with a distance between $0.8 \mathrm{~m}$ and $1.5 \mathrm{~m}$ from the geometry. At the lee side the location of the gauges is varied between $2 \mathrm{~m}$ and $6.5 \mathrm{~m}$ from the geometry. The results obtained for the wave amplitudes with this method are almost independent of the values of $\Delta x$ and $x_{1}$.

\section{Experimental results}

\subsection{The circular bodies}

Let us then consider the waves at the lee side of the circular bodies. In figures 1a-b we display measurements of $a_{+}^{(1)}$ and $a_{+}^{(2)}$ for the circle with radius $R=100 \mathrm{~mm}$ submerged with distance $h=100 \mathrm{~mm}$ between the free surface and its uppermost point. The scatter obtained by different runs are indicated in the figures. The figures show that $a_{+}^{(1)} / a$ always 
is very close to unity. The results for $a_{+}^{(2)} / a$ exhibit roughly a linear increase with $a$, which for small and moderate incoming wave amplitude agree with computations by second order potential theory (Friis, Grue and Palm 1991). We observe that the second harmonic wave amplitude $a_{+}^{(2)}$ is much smaller than $a_{+}^{(1)} . a_{+}^{(2)}$ is, however, much larger than the forced second harmonic wave amplitude, $a_{l+}^{(2)}$, which in all examples, within the accuracy of the experiments, agree with the values of $\frac{1}{2}\left(a_{+}^{(1)}\right)^{2} K$, also shown in the figures. The third and higher harmonic waves are very small in the examples.

Next we reduce the distance between the free surface and the uppermost point of the circle to $h=50 \mathrm{~mm}$. Measurements of $a_{+}^{(1)}$ and $a_{+}^{(2)}$ are shown in figure 2a for the circle with $R=100 \mathrm{~mm}$ and wave frequency $\omega=2 \pi \times 1.05 \mathrm{~Hz}$, in figure $2 \mathrm{~b}$ for $R=100 \mathrm{~mm}$ and $\omega=2 \pi \times 1.22 \mathrm{~Hz}$ and in figure $2 \mathrm{c}$ for the larger circle with $R=190 \mathrm{~mm}$ and $\omega=2 \pi \times 1.05 \mathrm{~Hz}$. $a_{+}^{(3)}, a_{+}^{(4)}, \ldots$, are again very small.

The values of the second harmonic free wave amplitude show some interesting features in these examples. We observe that $a_{+}^{(2)} / a$ is a monotonously growing quantity up to a maximum which occurs in the vicinity of the spilling limit, which also is indictated in the figures. We remark that the measurements of $a_{+}^{(2)}$ fit well with results from second order potental theory for small incoming wave amplitude. The maximum value of $a_{+}^{(2)} / a$ is, surprisingly, very close to 0.4 in these examples (figures $2 \mathrm{a}$ and $2 \mathrm{c}$ ) which means that up to $8 \%$ of the incoming energy flux is transferred to the second harmonic free waves. We also note that the maximum value of $a_{+}^{(2)}$ is almost $50 \%$ of the first harmonic transmitted wave amplitude. In other words, the magnitude of the second harmonic free wave amplitude may be comparable to the first harmonic wave amplitude in these examples. For larger incoming waves $a_{+}^{(2)} / a$ is a monotonous decaying function. The second harmonic free wave amplitude is completely dominating the forced second harmonic wave amplitude in these examples, i.e. $a_{+}^{(2)}>>a_{l_{+}}^{(2)}$.

In figure $2 \mathrm{a}$ we have also displayed theoretical results for $a_{+}^{(2)}$ obtained by Cointe (1989) who exploit potential theory with the exact nonlinear free surface condition. Cointe's computations are performed for $\omega=2 \pi H z$, a slightly smaller frequency than ours. The theoretical results for $a_{+}^{(2)}$ fit surprisingly well with the present measurements, even also when spilling or plunging is observed in the wave flume.

We observe that the values of $a_{+}^{(1)} / a$ exhibit a pronounced decay with increasing incoming amplitude, with the largest decay occurring for the largest cylinder. The generation of the second harmonic wave accounts for up to $8 \%$ of the incoming energy flux in these examples, and is, in addition to energy loss due to wave breaking, partly explaining the reduced values of $a_{+}^{(1)} / a$ in figures $2 \mathrm{a}$-c. In addition to these two effects, there is energy lost in the bodies' boundary layer, which obviously takes place more strongly at the larger cylinder, with the larger decay in $a_{+}^{(1)} / a$, than at the smaller cylinder, with a weaker decay in $a_{+}^{(1)} / a$, since the transfer of energy to the second harmonic wave and loss of energy due to wave breaking are approximately of the same strength in all of the three examples.

In the next examples, figures $3 \mathrm{a}-\mathrm{b}$, we consider the smaller circle situated very close to the free surface with $h=25 \mathrm{~mm}$. The results for $a_{+}^{(1)}$ and $a_{+}^{(2)}$ are similar to the former cases shown in figures $2 \mathrm{a}-\mathrm{b}$. The maximum of $a_{+}^{(2)} / a$ is now occurring for a smaller incoming wave amplitude, $a \simeq 5 \mathrm{~mm}$, with a maximum value being, surprisingly, almost the same as for the deeper submerged circles. Another interesting result is that the experimental values of $a_{+}^{(2)}$ show excellent agreement with second order potential theory for very small incoming 
wave amplitudes, but strongly deviate from this theory for incoming wave amplitudes greater than $5 \mathrm{~mm}$ and up to the spilling limit. We would expect second order theory to be appropriate for obtaining $a_{+}^{(2)}$ up to the breaking limit. The experimental results indicate, however, that strong nonlinear effects are contributing to the generation of the second harmonic waves at the body, which are not included in the second order theory. As in the former examples, the third and higher harmonic waves have very small amplitudes.

One of the striking results for the circular cylinder is that linear potential theory gives a vanishing reflection coefficient (Dean 1948). Recently, it has been shown that also the second harmonic reflection coefficient is identically zero (McIver and McIver 1990, Friis, Grue and Palm 1991). Our measurements support these theoretical results. The measured first harmonic reflection power, $a_{-}^{(1)} / a$, is always less than 0.05 , which is the accuracy of the experiments, even if the waves are breaking at the body. With no first harmonic reflection, i.e. $a_{-}^{(1)} \simeq 0$, which gives that $a_{l-}^{(2)} \simeq 0$, the surface elevation at the weather side of the cylinder is approximately given by

$$
\begin{array}{r}
\eta(x, t)=a \cos (K x-\omega t+\delta)+a_{l}^{(2)} \cos 2(K x-\omega t+\delta) \\
+a_{-}^{(2)} \cos \left(K_{2} x+2 \omega t+\delta_{-}^{(2)}\right)+\text { higher harmonic components }
\end{array}
$$

It is easy to demonstrate that we may measure $a_{-}^{(2)}$ by (12) in this case. The measurements show that $a_{-}^{(2)}$ is even much smaller than $a_{-}^{(1)}$. We also find that $\left|\hat{\eta}_{-}^{(n)}\right|, n=3,4, \ldots$, are very small. Our experimental results are thus indicating that there are no first and higher harmonic reflected waves due to the submerged circular cylinder. This result is also found experimentally by Chaplin (1984) who considered circular cylinders with deeper submergence.

\subsection{The rectangular bottom topography}

Let us then consider the higher harmonic waves generated at the rectangle which has a horizontal extention, $2 L=500 \mathrm{~mm}$, along the wave channel, is extending across the channel and down to the bottom of the channel. The rectangle is submerged with a small distance $h$ between the free surface and its horizontal top, which in the present examples is chosen to be either $h=37.5 \mathrm{~mm}$ or $h=50 \mathrm{~mm}$. A large part of the incoming wave energy may be reflected by this body, depending on the incoming wave length, the length of the rectangle and its submergence. Surprisingly, however, the major part of the incoming wave energy is transmitted when $\lambda$ is shorter than about ten times the horizontal extention $2 L$, i.e. $\lambda>20 L$. This fact is illustrated in figure 4 where the transmission coefficient, obtained by linear theory (see $\S 4$ ), is shown for $h / 2 L=0.075,0.1$ and for $\lambda>4 L$.

Results for the following two sets of parameters are then considered; set I: $h=37.5 \mathrm{~mm}$ and $\omega=2 \pi \times 0.95 \mathrm{~Hz}$, i.e. $h / 2 L=0.075, \omega^{2} h / g=0.136$, and set II: $h=50 \mathrm{~mm}$ and $\omega=2 \pi \times 1.05 H z$, i.e. $h / 2 L=0.1, \omega^{2} h / g=0.22$. In both examples the theoretical reflection coefficient is almost vanishing. The experimental reflection power is, however, approximately 0.2 , which means that $4 \%$ of the incoming wave energy is reflected. We display in figures 5 and 6 results for the amplitudes of the transmitted first, second and third harmonic free waves as a function of the incoming wave amplitude. The wave lengths of the first, second and third harmonic components are for set $\mathrm{I}: \lambda=1.62 \mathrm{~m}, 0.43 \mathrm{~m}$ and $0.19 m$, respectively (figure 5), and for set II: $\lambda=1.37 m, 0.36 m$ and $0.16 m$, respectively (figure 6). We observe that the generation of the higher harmonic waves is more powerful 
in these examples than in the previous, and we remark the strong presence of the third harmonic free waves which were not generated in the other examples. The results for $a_{+}^{(1)}$ and $a_{+}^{(2)}$ are very similar to those for the circular bodies, with the exception, however, that the values of $a_{+}^{(1)}$ and $a_{+}^{(2)}$ are almost of equal magnitude at the spilling limit in these examples. Surprisingly, the third harmonic wave amplitude becomes even larger than $a_{+}^{(2)}$ at the spilling limit for parameter set I (figure 5), and may be as large as $60 \%$ of the incoming wave amplitude. Approximately $25 \%$ of the incoming energy flux is transferred to the super harmonic waves at the spilling limit in this case. For parameter set II (figure 6) the measured maximal value of $a_{+}^{(2)} / a$ is approximately 0.6 with $a_{+}^{(3)} / a$, however, being much smaller. The fourth and higher harmonic waves have in these examples wave lengths being shorter than $10 \mathrm{~cm}$. Measurements of such short waves are very complicated since the energy is quickly dissipated.

For comparison with the experiments we develop in $\$ 4$ a simplified nonlinear theory for the rectangular bottom topography, by taking into account nonlinear and dispersive effects in the shallow region above the rectangle with matching to linearized theory in the deep water. Results for $a_{+}^{(1)}, a_{+}^{(2)}$ and $a_{+}^{(3)}$, obtained by this theory, are also shown in figures 5 and 6 . The theoretical computations are performed for the nonbreaking regime and agree surprisingly well with the experiments except close to the breaking limit.

We observe that the dissipation of energy becomes more and more powerful with increasing wave amplitude. The dissipation in the boundary layer at the geometries, being laminar in the examples, is for the nth harmonic oscillation proportional to $(n \omega)^{5 / 2}$ times the amplitude of oscillation squared. Thus, the dissipation becomes more pronounced when the super harmonic oscillations are large, which explaines why we observe a stronger dissipation for the examples with the rectangular bottom topography than in the examples for the circles. Furthermore, the dissipation leads to smaller experimental values of $a_{+}^{(1)}, a_{+}^{(2)}$ and $a_{+}^{(3)}$ than predicted by the theory.

In order to more closely examine the deformation of the incoming waves we show in figures 7 and 8 photographs of the surface elevation above the rectangle at subsequent time instants. The photographs are made with a motorized drive with a time difference between the pictures being $P \simeq 0.35 \mathrm{~s}$. The wave propagation direction is from left to right in the pictures. The photographs exhibit how the long incoming wave with one well defined crest steepens at the left end of the shallow region above the bottom topography (figures 7a and $8 \mathrm{a})$. When the wave has moved to the right end, however, we clearly observe from figures $7 \mathrm{~b}-\mathrm{c}$ and $8 \mathrm{~b}$ that higher harmonic wave components are superposed on the longer wave.

In both examples above we measure only very weak higher harmonic reflected waves with amplitudes being smaller than the experimental error.

Other examples, which are not shown here, also conclude that pronounced higher harmonic waves are introduced at the lee side of the obstacle, while there are, practically speaking, no such waves at the weather side. This is also the case when there is a large reflected first harmonic wave.

\subsection{Saturation}

In all the examples above we find that the generation of the higher harmonic wave components takes place with increasing strength for growing wave amplitude up to when breaking occurs at the obstacle. For still increasing incoming wave amplitude the transfer of energy to the higher harmonic components reduces in power, and we find that the higher harmonic 
wave amplitudes become saturated. Remarkably, the saturation value of $a_{+}^{(2)}$ occurs in all the examples, but for parameter set I for the rectangle, for

$$
a_{+}^{(2)} K_{2} \sim 0.1
$$

For parameter set I the saturation value of $a_{+}^{(2)}$ is $a_{+}^{(2)} K_{2} \sim 0.05$ The saturation values of $a_{+}^{(3)}$ occur in the examples for the bottom topography for $a_{+}^{(3)} K_{3} \sim a_{+}^{(2)} K_{2}$. Thus, the wave slope of the superposed transmitted waves remain bounded and small.

\section{Nonlinear theory}

Let us then study a theoretical approach for the wave diffraction due to the rectangular bottom topography. Relevant for the experiments the fluid layer may be assumed to be of infinite depth. Furthemore, the fluid is assumed inviscid, incompressible and the motion irrotational. As above, let the horizontal extention of the rectangle be denoted by $2 L$, be submerged with a small distance $h$ below the free surface such that $h / 2 L$ is a small quantity, and be extending infinitely deep downwards in the fluid. Let us apply coordinates with the $x$-axis, as introduced above, in the mean free surface, the $y$-axis vertical upwards and with the origin $\mathrm{O}$ on the vertical symmetry line of the rectangle. The uppermost corners of the rectangle are then located at $x= \pm L, y=-h$. Let the incoming wave train with wave number $K$, frequency $\omega=\sqrt{g K}$ and amplitude $a$ be incident from $x=-\infty$. The amplitude $a$ is growing from zero for $t=0$ to a constant value $a_{0}$ during a time interval being much longer than the wave period $2 \pi / \omega$.

\subsection{Nonlinear flow in the shallow region}

The incoming waves are assumed to be long compared to the local depth in the shallow region above the topography, which means that the flow there is weakly dispersive. To account for both nonlinear and weakly dispersive effects we apply the nonlinear Boussinesq equations to the flow there, i.e. for $-L<x<L,-h<y<\eta$. Introducing the depth averaged horizontal velocity $u(x, t)$ in the shallow region, the Boussinesq equations may be written

$$
\begin{gathered}
\frac{\partial \eta}{\partial t}=-\frac{\partial}{\partial x}((h+\eta) u) \quad \text { (continuity equation) } \\
\frac{\partial u}{\partial t}+\frac{1}{2} \frac{\partial}{\partial x} u^{2}=-g \frac{\partial \eta}{\partial x}+\frac{h^{2}}{3} \frac{\partial^{3} u}{\partial^{2} x \partial t} \quad \text { (equation of motion) }
\end{gathered}
$$

The pressure in the fluid is given by

$$
p(x, y, t)=\rho g(\eta-y)-\rho \frac{\partial^{2} u}{\partial x \partial t}\left((\eta-y) h+\frac{1}{2}\left(\eta^{2}-y^{2}\right)\right)
$$

Thus, the flow in the shallow region above the topography is determined by the values of $\eta$ and $u$.

\subsection{Initial value problem for the flow outside the shallow region}

The flow at the weather side and the lee side of the rectangle, i.e. for $|x|>L$, may according to the previous assumptions be governed by a velocity potential $\phi$ which satisfies 
the two-dimensional Laplace equation

$$
\frac{\partial^{2} \phi}{\partial x^{2}}+\frac{\partial^{2} \phi}{\partial y^{2}}=0
$$

in the fluid domain. The potential $\phi$ is subject to the nonlinear free surface boundary condition. Assuming, however, that the wave slope of the incoming waves is small, i.e.

$$
a K<<1
$$

and that also the diffracted waves are of small wave slope, the free surface boundary condition may be linearized, i.e.

$$
\frac{\partial^{2} \phi}{\partial t^{2}}+g \frac{\partial \phi}{\partial y}=0, y=0,|x|>L
$$

At the vertical walls of the rectangle $\phi$ is subject to

$$
\frac{\partial \phi}{\partial x}=0, x= \pm L,-\infty<y<-h
$$

At the edges of the rectangle we enforce continuity of the volume flux, the free surface elevation and the depth averaged pressure. The first requirement gives

$$
\int_{-h}^{0} \frac{\partial \phi( \pm L, y, t)}{\partial x} d y=Q( \pm L, t)
$$

where we have introduced the volume flux at the ends of the shallow region by

$$
Q=u(\eta+h)
$$

As a first approximation, let us assume that $\partial \phi / \partial x$ is constant in the vertical coordinate. (23) then reduces to

$$
\frac{\partial \phi( \pm L, y, t)}{\partial x}=\frac{1}{h} Q( \pm L, t) \quad-h<y<0
$$

In addition to these conditions $\phi$ also fulfills the condition

$$
|\nabla \phi| \rightarrow 0 \quad y \rightarrow-\infty
$$

and the initial conditions that there is no free surface elevation and no applied pressure at the free surface for $t \leq 0$, i.e.

$$
\phi=\partial \phi / \partial t=0, \quad y=0, t=0
$$

\subsection{Solution of the initial value problem}

The solution of the boundary value problem (19)-(27) on the weather side of the rectangle (for $x<-L$ ) is appropriately composed by a standing wave potential $\phi_{0}$ and a velocity potential $\phi_{-L}$ due to the outflux at $x=-L$, i.e.

$$
\phi=\phi_{0}+\phi_{-L}
$$


The standing wave potential corresponding to an incoming and a reflected wave with amplitude $a$ and wave number $K=\omega^{2} / g$ reads

$$
\phi_{0}(x, y, t)=-2 \frac{a g}{\omega} \exp (K y) \cos K(x+L) \sin \left(\omega t+\delta_{0}\right)
$$

where $\delta_{0}$ is an arbitrary phase angle which may be set equal to zero without loss of generality. $a$ increases monotonously from zero to the constant value $a_{0}$ following

$$
a=a_{0}\left[\frac{1}{2}+\frac{1}{2} \tanh (\omega t-3)\right]
$$

The transient velocity potential $\phi_{-L}$ may be given by a source distribution with source strength $q_{-L}\left(y^{\prime}, t\right),-h<y^{\prime}<0$ at $x=-L$. By applying the velocity potential due to one pulsating source located at a point $\left(-L, y^{\prime}\right)$ in the fluid domain satisfying (19) (except at $\left.x=-L, y=y^{\prime}\right),(21),(22),(26),(27)$, which is given by Wehausen and Laitone (1960, eq. 13.54), $\phi_{-L}$ reads

$$
\begin{array}{r}
\phi_{-L}(x, y, t)=\frac{1}{2 \pi} \int_{-h}^{0} q_{-L}\left(y^{\prime}, t\right) \ln \frac{r}{r_{1}} d y^{\prime} \\
-\frac{g}{\pi} \int_{-h}^{0} d y^{\prime} \int_{0}^{t} d \tau q_{-L}\left(y^{\prime}, \tau\right) \int_{0}^{\infty} \frac{d k}{\sqrt{g k}} \sin (\sqrt{g k}(t-\tau)) \exp k\left(y+y^{\prime}\right) \cos k(x+L)
\end{array}
$$

where $r=\left|x+L+i\left(y-y^{\prime}\right)\right|$ and $r_{1}=\left|x+L+i\left(y+y^{\prime}\right)\right|$ The horizontal velocity for $x<-L$ is then given by $\partial\left(\phi_{0}+\phi_{-L}\right) / \partial x$. By applying (31), and letting $x \rightarrow-L$ from below we obtain for $-h<y<0$

$$
\frac{\partial\left(\phi_{0}+\phi_{-L}\right)}{\partial x} \rightarrow-\frac{1}{2} q_{-L}(y, t)
$$

The source strength at $x=-L$ is then determined by applying (25), which gives

$$
q_{-L}(y, t)=-\frac{2}{h} Q(-L, t),-h<y<0
$$

The velocity potential $\phi_{-L}$ for $x<-L$ is thus given by

$$
\begin{array}{r}
\phi_{-L}(x, y, t)=-\frac{Q(-L, t)}{\pi h} \int_{-h}^{0} \ln \frac{r}{r_{1}} d y^{\prime} \\
+\frac{2 g}{\pi h} \int_{0}^{t} d \tau Q(-L, \tau) \int_{0}^{\infty} \frac{d k}{\sqrt{g k^{3}}} \sin (\sqrt{g k}(t-\tau))(1-\exp (-k h)) \exp (k y) \cos k(x+L)
\end{array}
$$

where in the last term we have carried out the vertical integration.

The potential $\phi_{L}$ governing the flow on the lee side of the rectangle, i.e. for $x>L$, may correspondingly be given by a source distribution $q_{L}(y, t)$ at $x=L,-h<y<0$, i.e.

$$
\begin{array}{r}
\phi_{L}(x, y, t)=\frac{1}{2 \pi} \int_{-h}^{0} q_{L}\left(y^{\prime}, t\right) \ln \frac{r}{r_{1}} d y^{\prime} \\
-\frac{g}{\pi} \int_{-h}^{0} d y^{\prime} \int_{0}^{t} d \tau q_{L}\left(y^{\prime}, \tau\right) \int_{0}^{\infty} \frac{d k}{\sqrt{g k}} \sin (\sqrt{g k}(t-\tau)) \exp k\left(y+y^{\prime}\right) \cos k(x-L)
\end{array}
$$

where $r=\left|x+L+i\left(y-y^{\prime}\right)\right|$ and $r_{1}=\left|x+L+i\left(y+y^{\prime}\right)\right|$. By taking the derivative of (35) with respect to $x$ and letting $x \rightarrow L$ from above we obtain

$$
\frac{\partial \phi_{L}}{\partial x} \rightarrow \frac{1}{2} q_{L}(y, t)
$$


The source strength at $x=L$ is then, from (25), obtained by

$$
q_{L}(y, t)=\frac{2}{h} Q(L, t),-h<y<0
$$

Thus

$$
\begin{array}{r}
\phi_{L}(x, y, t)=\frac{Q(L, t)}{\pi h} \int_{-h}^{0} \ln \frac{r}{r_{1}} d y^{\prime} \\
-\frac{2 g}{\pi h} \int_{0}^{t} d \tau Q(L, \tau) \int_{0}^{\infty} \frac{d k}{\sqrt{g k^{3}}} \sin (\sqrt{g k}(t-\tau))(1-\exp (-k h)) \exp (k y) \cos k(x-L)
\end{array}
$$

At the edges of the rectangle we enforce, in addition to continuity of the volume flux, continuity in the free surface elevation and in the depth averaged pressure. This involves evaluation of $\partial \phi / \partial t$ at $x= \pm L$ which is obtained from (29), (34) and (38). For $\partial \phi_{-L} / \partial t$ at $x=-L$ and $\partial \phi_{L} / \partial t$ at $x=L$ we have

$$
\begin{array}{r}
\frac{\partial \phi_{-L}(-L, y, t)}{\partial t}=-\frac{\dot{Q}(-L, t)}{\pi h} \int_{-h}^{0} \ln \frac{\left|y-y^{\prime}\right|}{\left|y+y^{\prime}\right|} d y^{\prime} \\
+\frac{2 g}{\pi h} \int_{0}^{t} d \tau Q(-L, \tau) \int_{0}^{\infty} \frac{d k}{k} \cos (\sqrt{g k}(t-\tau))(1-\exp (-k h)) \exp (k y) \\
\frac{\partial \phi_{L}(L, y, t)}{\partial t}=\frac{\dot{Q}(L, t)}{\pi h} \int_{-h}^{0} \ln \frac{\mid y-y^{\prime}}{\left|y+y^{\prime}\right|} d y^{\prime} \\
-\frac{2 g}{\pi h} \int_{0}^{t} d \tau Q(L, \tau) \int_{0}^{\infty} \frac{d k}{k} \cos (\sqrt{g k}(t-\tau))(1-\exp (-k h)) \exp (k y)
\end{array}
$$

where $\dot{Q}=d Q / d t$.

\subsection{The far-field solution for $t \rightarrow \infty$}

The super harmonic oscillations introduced at the bottom topography give that the volume flux at the corners, for $t \rightarrow \infty$, behave as

$$
Q( \pm L, t)=\operatorname{Re} \sum_{n \geq 1} Q_{ \pm L}^{(n)} \exp (i n \omega) t+O(1 / t)
$$

The velocity potentials $\phi_{ \pm L}$ become accordingly

$$
\phi_{ \pm L}(x, y, t)= \pm \operatorname{Re}\left\{\sum_{n \geq 1} \frac{2}{h} Q_{ \pm L}^{(n)} \exp (i n \omega t) \int_{-h}^{0} G^{(n)}\left(x, y, \pm L, y^{\prime}\right) d y^{\prime}\right\}+O(1 / t)
$$

where

$$
G^{(n)}\left(x, y, \pm L, y^{\prime}\right)=\frac{1}{2 \pi} \ln \frac{r}{r_{1}}-\frac{1}{\pi} \int_{0}^{\infty} \frac{\exp k\left(y+y^{\prime}\right) \cos k(x \mp L)}{k-K_{n}} d k
$$

denotes the familiar frequency domain Green function due to a point source oscillating with frequency $n \omega, n=1,2,3, \ldots$. The integration is above the pole $K_{n}=n^{2} \omega^{2} / g=n^{2} K$. For $x \rightarrow \pm \infty$ we have

$$
G^{(n)}=i \exp \left[K_{n}\left(y+y^{\prime} \mp i(x \mp L)\right)\right] \quad x \rightarrow \pm \infty
$$


Thus, by carrying out the vertical integration over the gaps at the edges of the rectangular bottom topography, the free surface elevation for $t \rightarrow \infty, x \rightarrow \pm \infty$, is obtained as

$$
\begin{gathered}
\eta(x, t)=\operatorname{Re} \sum_{n \geq 1} A_{+}^{(n)} \exp i\left(n \omega t-K_{n}(x-L)\right) \quad x \rightarrow \infty \\
\eta(x, t)=\operatorname{Re}\left\{a \exp i(\omega t-K(x+L))+\sum_{n \geq 1} A_{-}^{(n)} \exp i\left(n \omega t+K_{n}(x+L)\right)\right\} \quad x \rightarrow-\infty
\end{gathered}
$$

where

$$
\begin{aligned}
A_{+}^{(n)} & =\frac{2 Q_{L}^{(n)}}{n \omega h}\left(1-\exp \left(-K_{n} h\right)\right) \\
A_{-}^{(n)} & =-\frac{2 Q_{-L}^{(n)}}{n \omega h}\left(1-\exp \left(-K_{n} h\right)\right)+\delta_{n 1} a
\end{aligned}
$$

and $\delta_{n 1}$ denotes the Dirac delta function.

\section{Numerical integration and examples}

Let the domain $|x| \leq L$ be subdivided into $N+1$ segments with equal length $\Delta x=\frac{2 L}{N+1}$ and let $\Delta t$ denote a constant time step. Following Pedersen (1991) the Boussinesq equations are integrated by the following scheeme which is staggered in both time and space

$$
\begin{gathered}
{\left[\delta_{t} \eta=-\delta_{x}\left\{\left(1+\bar{\eta}^{x t}\right) u\right\}\right]_{i}^{\left(k+\frac{1}{2}\right)}} \\
{\left[\delta_{t} u+\delta_{x} T=-\delta_{x} g \eta+\frac{h^{2}}{3} \delta_{x}^{2} \delta_{t} u\right]_{i+\frac{1}{2}}^{(k)}}
\end{gathered}
$$

where []$_{i}^{(k)}$ denotes the value at $x_{i}=-L+i \Delta x$ and time $t_{k}=k \Delta t$, and

$$
T_{i}^{(k)}=\frac{1}{2}\left(\bar{u}^{x}\right)_{i}^{\left(k-\frac{1}{2}\right)}\left(\bar{u}^{x}\right)_{i}^{\left(k+\frac{1}{2}\right)}
$$

We have introduced the symmetric difference operator, $\delta_{t}$, and the midpoint average operator, $(\bar{l})$, of a quantity $f(x, t)$, defined by

$$
\begin{aligned}
{\left[\delta_{t} f\right]_{i}^{(k)} } & \equiv \frac{1}{\Delta t}\left[f\left(x_{i}, t_{k+\frac{1}{2}}\right)-f\left(x_{i}, t_{k-\frac{1}{2}}\right)\right] \\
{\left[\bar{f}^{t}\right]_{i}^{(k)} } & \equiv \frac{1}{2}\left[f\left(x_{i}, t_{k+\frac{1}{2}}\right)+f\left(x_{i}, t_{k-\frac{1}{2}}\right)\right]
\end{aligned}
$$

The operators $\delta_{x}$ and ()$^{x}$ are defined accordingly. We thus obtain the values of $u$ at $x_{i+\frac{1}{2}}, i=0,1, \ldots, N$ for $t_{k+\frac{1}{2}}=\left(k+\frac{1}{2}\right) \Delta t$ and the values of $\eta$ at $x_{i}, i=1, \ldots, N$ for $t_{k+1}=$ $(k+1) \Delta t$. The volume flux at $x= \pm L$ is obtained by extrapolation, i.e.

$$
\begin{aligned}
Q\left(L, t_{k+\frac{1}{2}}\right) & =\left(\frac{3}{2} u_{N}^{\left(k+\frac{1}{2}\right)}-\frac{1}{2} u_{N-1}^{\left(k+\frac{1}{2}\right)}\right) \eta_{N+1}^{\left(k+\frac{1}{2}\right)} \\
Q\left(-L, t_{k+\frac{1}{2}}\right) & =\left(\frac{3}{2} u_{0}^{\left(k+\frac{1}{2}\right)}-\frac{1}{2} u_{1}^{\left(k+\frac{1}{2}\right)}\right) \eta_{0}^{\left(k+\frac{1}{2}\right)}
\end{aligned}
$$


The free surface elevation and the integrated pressure at $x_{0}=-L$ and $x_{N+1}=L$ are obtained by (29), (39) and (40). The numerical procedure of integrating $\partial \phi_{ \pm L} / \partial t$ follows Yeung (1982), with the time integrals discretized by

$$
\begin{array}{r}
\int_{0}^{t_{k}} d \tau Q( \pm L, \tau) \int_{0}^{\infty} \frac{d k}{k} \cos \left(\sqrt{g k}\left(t_{k}-\tau\right)(1-\exp (-k h) \exp (k y)\right. \\
=\sum_{m=0}^{k-1} Q\left( \pm L, t_{m+\frac{1}{2}}\right) \int_{t_{m}}^{t_{m+1}} d \tau \int_{0}^{\infty} \frac{d k}{k} \cos \left(\sqrt{g k}\left(t_{k}-\tau\right)(1-\exp (-k h) \exp (k y)\right. \\
=\sum_{m=0}^{k-1}\left(Q\left( \pm L, t_{m+\frac{1}{2}}\right)-Q\left( \pm L, t_{m-\frac{1}{2}}\right)\right) \times \\
\times \int_{0}^{\infty} \frac{d k}{\sqrt{g k^{3}}} \sin \left(\sqrt{g k}\left(t_{k}-t_{m}\right)(1-\exp (-k h) \exp (k y)\right. \\
=\Delta t \sum_{m=0}^{k-1} \dot{Q}\left( \pm L, t_{m}\right) \int_{0}^{\infty} \frac{d k}{\sqrt{g k^{3}}} \sin \left(\sqrt{g k}\left(t_{k}-t_{m}\right)(1-\exp (-k h) \exp (k y)\right.
\end{array}
$$

Now, from Yeung (1982) we have

$$
\begin{array}{r}
\int_{0}^{\infty} \frac{d k}{\sqrt{g k^{3}}} \sin \sqrt{g k} t(1-\exp (k y)) \\
=\frac{d}{d t} \int_{0}^{t} d \tau \int_{0}^{\infty} \frac{d k}{\sqrt{g k^{3}}} \sin \sqrt{g k t} t(1-\exp (k y))=2 t \mathcal{F}_{2}\left(\frac{1}{2} t \sqrt{g /|y|}\right)
\end{array}
$$

where

$$
\mathcal{F}_{2}(\Omega)=\int_{\Omega}^{\infty} \frac{\mathcal{F}(u)}{u^{2}} d u
$$

Here $\mathcal{F}(u)$ denotes Dawson's integral given by

$$
\mathcal{F}(u)=\exp \left(-u^{2}\right) \int_{0}^{u} \exp \left(\alpha^{2}\right) d \alpha
$$

which may effectively be computed, see Newman (1987). Introducing (55) with (56) into (39) and (40) we obtain

$$
\begin{array}{r}
\frac{\partial \phi_{ \pm L}( \pm L, y, t)}{\partial t}= \pm \frac{\dot{Q}\left( \pm L, t_{k}\right)}{\pi h} \int_{-h}^{0} \ln \frac{\left|y-y^{\prime}\right|}{\left|y+y^{\prime}\right|} d y^{\prime} \\
\mp \frac{4 g}{\pi h}(\Delta t)^{2} \sum_{m=0}^{k-1}(k-m) \dot{Q}\left( \pm L, t_{m}\right)\left[\mathcal{F}_{2}\left(\frac{t_{k}-t_{m}}{2} \sqrt{g /|y-h|}\right)-\mathcal{F}_{2}\left(\frac{t_{k}-t_{m}}{2} \sqrt{g /|y|}\right)\right]
\end{array}
$$

We remark that the memory terms involve the time differentiated volume fluxes at the previous time steps.

The numerical integration of the Boussinesq equations with the end conditions at $x=$ $\pm L$ is for the linear case, i.e. $a / h \rightarrow 0$, compared with the corresponding time domain solution, with excellent agreement.

Convergence of the numerical scheeme is achieved by increasing the value of $N$ and decreasing the time step $\Delta t$. We find that the simulations have converged for $N=O(100)$ and $\Delta t \sqrt{g / h}=O(0.1)$, giving the integrated quantities with at least three significant digits. No smoothing is applied. 


\begin{tabular}{|l|l|l|l|l|l|l|}
\hline$a / h$ & $\frac{\left|Q^{(1)}\right|}{a \sqrt{g h}}$ & $\frac{Q^{(2)} \mid}{a \sqrt{g h}}$ & $\frac{Q^{(3)} \mid}{a \sqrt{g h}}$ & $\frac{\mid Q^{(4)}}{a \sqrt{g h}}$ & $\frac{\mid Q^{(5)}}{a \sqrt{g h}}$ & $\frac{\left|Q^{(6)}\right|}{a \sqrt{g h}}$ \\
\hline 0 & 1.460 & 0 & 0 & 0 & 0 & 0 \\
\hline 0.1 & 1.347 & 0.425 & 0.318 & 0.122 & 0.046 & 0.019 \\
\hline 0.136 & 1.261 & 0.471 & 0.501 & 0.240 & 0.114 & 0.064 \\
\hline
\end{tabular}

Table 1: Fourier components of volume flux $Q(L, t)$ for $a / h=0,0.1,0.136, \omega^{2} h / g=$ $0.136, h / 2 L=0.075$.

\begin{tabular}{|l|l|l|l|l|l|l|}
\hline$a / h$ & $\left|A_{+}^{(1)}\right| / a$ & $\left|A_{+}^{(2)}\right| / a$ & $\left|A_{+}^{(3)}\right| / a$ & $\left|A_{+}^{(4)}\right| / a$ & $\left|A_{+}^{(5)}\right| / a$ & $\left|A_{+}^{(6)}\right| / a$ \\
\hline 0 & 1.007 & 0 & 0 & 0 & 0 & 0 \\
\hline 0.1 & 0.929 & 0.484 & 0.406 & 0.147 & 0.048 & 0.017 \\
\hline 0.136 & 0.870 & 0.536 & 0.639 & 0.289 & 0.120 & 0.058 \\
\hline
\end{tabular}

Table 2: Far-field amplitudes for $a / h=0,0.1,0.136, \omega^{2} h / g=0.136, h / 2 L=0.075$.

\subsection{Numerical results}

The numerical model is then applied to the same sets of parameters as for the experiments. In figures $9 a-b$ are displayed the free surface elevation at two subsequent time instants for three different ratios between the incoming wave amplitude and the local water depth, $a / h=0,0.1,0.136$, for the parameters in set I, i.e. $h / 2 L=0.075, \omega^{2} h / g=0.136$. The ratio $a / h=0.136$ corresponds to the limit where spilling is observed in the experiments. The figures clearly show that the wave steepens with increasing value of $a / h$. Furthermore, we observe that the presence of shorter waves superposed on the basic mode takes place more strongly with increasing incoming wave amplitude. These two features are also emphasized in figure 10 which displays the volume flux at $x=L$ as a function of time. We display furthermore in table 1 the six first Fourier components of $Q(L, t)$, which shows that there are significant interactions up to the fifth time harmonic for the largest incoming wave amplitude. We note the strong presence of the second and the third harmonic oscillations in the volume flux for $a / h=0.136$. The higher harmonic components of the volume flux at $x=L$ are generating the free super harmonic waves in the far-field, with amplitudes given by (47). In table 2 the far-field amplitudes corresponding to the volume fluxes from table 1 are obtained, illustrating that the same number of super harmonic oscillations in the far-field and the near-field are observed. The volume flux $Q(-L, t)$ at the left end of the rectangle shows, in contrast to $Q(L, t)$, almost no signs of higher harmonic oscillations, even for the larger incoming wave amplitudes. This explains that the higher harmonic waves at the weather side of the rectangle have practically speaking vanishing amplitudes.

It is also of interest to compare the computed free surface elevation with the photographs shown in section 3. The non-dimensional time difference between the pictures is for parameter set I, $P \sqrt{g / h} \simeq 5.7(h=3.75 \mathrm{~cm})$, and for parameter set II, $P \sqrt{g / h} \simeq 4.9(h=5 \mathrm{~cm})$. Numerical results for the surface elevation in natural scale at time instants corresponding to the photographs are displayed in figures $11 \mathrm{a}-\mathrm{c}$ and $12 \mathrm{a}-\mathrm{b}$. The agreement between the photos and the numerical results of $\eta$ is striking, illustrating that the theoretical model gives a relevant representation of the flow in the shallow region. 
We have also made some numerical simulations by neglecting the dispersion in the shallow region, with results shown in figures 13a-c. The nonlinear Boussinesq equations are then reduced to the nonlinear Airy equations. The Airy equations, which are integrated by the two-stage Lax-Wendroff scheeme (see Fletcher 1988, p. 353), give a solution which differs from the solution by the Boussinesq equations and from what we observe in the experiments in two respects: Firstly, the surface elevation becomes steeper, and secondly, the wave crest moves with a larger speed than given by the Boussinesq equations and than observed in the experiments. We also observe that the steep wave predicted by Airy equations leads to reflection of shorter waves at $x=L$, as seen in figure 13c. The matching procedure is, however, very effective when the effect of dispersion is included as in the Boussinesq equations, leading to a wave profile being less steep. For small values of $a / h$, when the generation of the shorter waves is weaker and the effect of dispersion is correspondingly small, the Airy equations and the Boussinesq equations give, however, coinciding results.

As a check of the computations we invoke the mean energy flux at a vertical control surface extending from the bottom of the fluid layer to the free surface, which at a location $x$ is given by

$$
R_{x}=\overline{\int_{\text {bottom }}^{\eta}\left(p+\frac{1}{2} \rho|\vec{v}|^{2}+\rho g y\right) u d y}
$$

Hefe $\vec{v}$ denotes the velocity vector in the fluid and a bar the time average. In the shallow region, where the pressure is given by (18) and $|\vec{v}|^{2} \simeq u^{2}$, we obtain

$$
R_{x}=\overline{\rho u(h+\eta)\left(g \eta+\frac{1}{2} u^{2}-\frac{1}{3}(h+\eta)^{2} \frac{\partial^{2} u}{\partial t \partial x}\right)}
$$

For $t \rightarrow \infty$ the mean energy flux at $x=-\infty$ is given by

$$
R_{-\infty}=E_{0} c_{g}^{(1)}-\sum_{n \geq 1} E_{-}^{(n)} c_{g}^{(n)}
$$

and at $x=\infty$ by

$$
R_{\infty}=\sum_{n \geq 1} E_{+}^{(n)} c_{g}^{(n)}
$$

where $E_{0}=\frac{1}{2} \rho g a^{2}, E_{-}^{(n)}=\frac{1}{2} \rho g\left|A_{-}^{(n)}\right|^{2}$ and $E_{+}^{(n)}=\frac{1}{2} \rho g\left|A_{+}^{(n)}\right|^{2}$ denote the mean energy densities of the incoming, reflected and transmitted waves, respectively. $c_{g}^{(n)}=\frac{1}{2} g / n \omega, n=$ $1,2, \ldots$, denote the corresponding group velocities of the incoming and scattered waves.

The mean energy flux is almost constant in the linear case, as seen in figure 14 . The energy flux has, on the other hand, a small variation along the $x$-coordinate, and a weak, total increase of up to $7 \%$ of the incoming energy flux across the shallow region for the largest value of $a / h$. The oscillations in the energy flux indicate the accuracy of the theoretical model.

\section{Conclusion}

We have experimentally and theoretically studied incoming deep water waves with small wave slope propagating over a slightly submerged cylindrical body or a bottom topography being close to the free surface. The wave length is much greater than the local water depth above the obstacle. Nonlinear free surface effects at the body or bottom topography 
introduce asymmetry and skewness to the initially symmetric wave profile, and generate a hierarchy of shorter super harmonic free waves propagating away from the obstacle and superposed upon the transmitted and reflected waves with wave length being equal to the incoming wave length. The super harmonic waves may attain prominent amplitudes at the obstacle's lee side. This is, however, not true at the weather side of the obstacle where the super harmonic waves are very small. The generation of the higher harmonic waves at the lee side become more and more powerful with increasing incoming wave amplitude up to when breaking occurs at the obstacle. The amplitudes of the second and third harmonic waves attain at the breaking limit maximal values compared to the incoming wave amplitude, and are in some examples found to be of the same order of magnitude as the first harmonic transmitted waves. We find in the present examples that up to about $25 \%$ of the incoming energy flux may be transferred to the super harmonic free waves at the lee side of the obstacle.

The theoretical model, developed for a rectangular bottom topography with a horizontal top, accounts for nonlinearity by the Boussinesq equations in the shallow region above the bottom topography, with matching at the edges of the topography to linearized potential theory applied in the deep water regions at the weather side and the lee side. The theoretical and experimental results for the local flow at the bottom topography and in the far-field show good agreement for non-breaking waves. The theoretical model illustrates that the nonlinear effects at the obstacle introduce interactions between several super harmonic wave components locally, generating super harmonic free waves accordingly. Furthermore, the theory empasizes that both nonlinearity and dispersion are important at the obstacle, while dispersion is the dominating effect for the flow far away. The matching procedure, applied between the linear and nonlinear flows, is very effective in the present examples.

Nonlinear resonant interaction may occur between the generated first, second and third harmonic free waves when their wave frequencies and wave numbers are forming tetrads by (resonant triads are impossible)

$$
\begin{aligned}
& -\omega+2 \omega+2 \omega-3 \omega=0 \\
& K+K_{2}+K_{2}-K_{3}=0
\end{aligned}
$$

This interaction is, however, of fourth order in the wave steepness, being very weak in the examples considered.

\section{Acknowledgements}

The author acknowlegdes Dr. Geir Pedersen for providing the Boussinesq equation solver, and the assistance by Arve Kvalheim, Øyvind Strand and Svein Vesterby with the experimental set up. Preliminary results were presented at the 3rd and 4th Intnl. Workshops on Water Waves and Floating Bodies held respectively at Woods Hole, Massachusetts, USA, 1988, and Øystese, Norway, 1989. The theoretical part of this research was initiated while the author was visiting at Dept. of Ocean Engineering, MIT, USA, 1987-88, kindly sponsored by Jansons Legat, the Royal Norwegian Council for Science and the Humanities (NAVF) and the Scandinavian American Association. 


\section{References}

[1] Chaplin, J. R., Nonlinear forces on a horizontal cylinder beneath waves. J. Fluid Mech. 147, pp. 449-4641984.

[2] Cointe, R., Nonlinear simulation of transient free surface flows. 5th International Confer. on Numerical Ship Hydrodynamics, 1989.

[3] Dean, W. R., On the reflection of surface waves by a circular cylinder. Proc. Camb. Phil. Soc. 441948.

[4] Fletcher, C. A. J., Computational Techniques for Fluid Dynamics I. Springer Series in Computational Physics. 1988.

[5] Friss, A., Grue, J. and Palm, E., Application of Fourier transform to the second order 2D wave diffraction problem. M. P. Tulin's Festshrift: Mathematical Approaches in Hydrodynamics, SIAM Publ. Philadelphia, Pennsylvania. 1991.

[6] Grue, J. and Granlund, K., Impact of nonlinearity upon waves travelling over a submerged cylinder. Abstr. 3rd Intl. Workshop on Water Waves and Floating Bodies, Woods Hole, Mass. 1988.

[7] Kit, L., Shemer, L. And Miloh, T., Experimental and theoretical Finite difference representations of nonlinear waves. J. Fluid Mech 181, pp. 265-291 1987.

[8] LeE, C. M., The second order theory of heaving cylinders in a free surface. J. Ship Res. 12, pp. 313-327 1968.

[9] Longuett-Higgins, M. S., The mean forces exerted by waves on floating or submerged bodies with applications to sand bars and wave power mashines. Proc. $R$. Soc. Lond. A. 352, pp. 463-480 1977.

[10] McIver, M. AND McIver, P., Second-order wave diffraction by a submerged circular cylinder. J. Fluid Mech 219, pp. 519-529 1990.

[11] Pedersen, G. K., Finite difference representations of nonlinear waves. Int. J. of Numerical methods in fluids 1991 (In press).

[12] Newman, J. N., Evaluation of the wave resistance Green Function: Part 2 - The single integral on the centerplane. J. Ship Res. 31, pp. 145-150 1987.

[13] VADA, T., A numerical solution of the second-order wave-diffraction problem for a submerged cylinder of arbitrary shape. J. Fluid Mech. 174, pp. 23-37 1987.

[14] Wehausen, J. V. and Laitone, E. V., Surface waves. Handbuch der Physik IX 1960.

[15] Williams, A nonlinear water wave problem. Ph.D.thesis, Dept. of Naval Arch. E Offsh. Engg, Univ. Calif, Berkeley 1964.

[16] Yeung, R. W., Transient heaving motion of floating cylinders. J. Engg. Math. 16, pp. 97-119 1982 . 


\section{Figure captions}

Figure 1.

Measurements of first and second harmonic free wave amplitudes at the lee side of the circular cylinder vs. incoming wave amplitude. $h=100 \mathrm{~mm}$. Squares: $a_{+}^{(1)} / a$, triangles: $a_{+}^{(2)} / a$, dotted line: $a_{l+}^{(2)} \simeq \frac{1}{2}\left(a_{+}^{(1)}\right)^{2} K / a$, dashed line: $a_{+}^{(2)} / a$ obtained by second order theory (Friis et al. 1991). a) small cylinder $(R=100 \mathrm{~mm}), \omega=2 \pi \times 1.05 \mathrm{~Hz}$. b) small cylinder $(R=100 \mathrm{~mm}), \omega=2 \pi \times 1.22 \mathrm{~Hz}$.

\section{Figure 2.}

Same as figure 1 , but $h=50 \mathrm{~mm}$. a) small cylinder $(R=100 \mathrm{~mm}), \omega=2 \pi \times 1.05 \mathrm{~Hz}$. Crosses: $a_{+}^{(2)} / a$ obtained by nonlinear theory, Cointe $(1989$, figure 12$)$. b) small cylinder $(R=100 \mathrm{~mm}), \omega=2 \pi \times 1.22 \mathrm{~Hz} . \mathrm{c})$ large cylinder $(R=190 \mathrm{~mm}), \omega=2 \pi \times 1.05 \mathrm{~Hz}$. The arrows denote respectively spilling $(\mathrm{S})$ and plunging $(\mathrm{P})$ limits.

Figure 3.

Same as figure 1 , but $h=25 \mathrm{~mm}$. a) small cylinder $(R=100 \mathrm{~mm}), \omega=2 \pi \times 1.05 \mathrm{~Hz}$. b) small cylinder $(R=100 \mathrm{~mm}), \omega=2 \pi \times 1.22 \mathrm{~Hz}$. The arrows denote respectively spilling $(\mathrm{S})$ and plunging $(\mathrm{P})$ limits.

Figure 4.

Linear transmission coefficient $a_{+}^{(1)} / a$ for the rectangular bottom topography vs. $2 L / \lambda$, obtained by the theory described in $\S 4$. Solid line: $h / 2 L=0.075$, dashed line: $h / 2 L=0.1$.

Figure 5.

First, second and third harmonic free wave amplitudes at the lee side of the rectangular bottom topography, vs. $a$, for parameter set I, i.e. $h=37.5 \mathrm{~mm}, \omega=2 \pi \times 0.95 \mathrm{~Hz}$. Measurements: Squares: $a_{+}^{(1)} / a$, triangles: $a_{+}^{(2)} / a$, diamonds: $a_{+}^{(3)} / a$. Nonlinear theory: Solid line: $a_{+}^{(1)} / a$, dashed line: $a_{+}^{(2)} / a$, dotted line: $a_{+}^{(3)} / a$. The arrows denote respectively spilling $(\mathrm{S})$ and plunging $(\mathrm{P})$ limits.

Figure 6.

Same as figure 5, but parameter set II, i.e. $h=50 \mathrm{~mm}, \omega=2 \pi \times 1.05 \mathrm{~Hz}$.

Figure 7.

Photographs of the wave profile at the rectangular bottom topography for $h=37.5 \mathrm{~mm}, \omega=$ $2 \pi \times 0.95 \mathrm{~Hz}, a=5.1 \mathrm{~mm}(a / h=0.136)$. a) time instant $\left.t_{1}, \mathrm{~b}\right)$ time instant $\left.t_{1}+0.35 \mathrm{sec}, \mathrm{c}\right)$ time instant $t_{1}+0.70 \mathrm{sec}$.

\section{Figure 8.}

Photographs of the wave profile at the rectangular bottom topography for $h=50 \mathrm{~mm}, \omega=$ $2 \pi \times 1.05 \mathrm{~Hz}, a=7.5 \mathrm{~mm}(a / h=0.15)$. a) time instant $\left.t_{2}, \mathrm{~b}\right)$ time instant $t_{2}+0.35 \mathrm{sec}$.

Figure 9.

Surface elevation in the shallow region above the rectangular bottom topography. $h / 2 L=$ $0.075, \omega^{2} h / g=0.136$. Numerical simulations with $N=100, \Delta t \sqrt{g / h}=0.1$. Solid line: 
$a / h=0.136$, dashed line: $a / h=0.1$, dotted line: $a / h=0$. a) Time instant $t_{1}$ corresponding to the photograph shown in figure $7 \mathrm{a}$. b) Time instant $t_{1}+0.35 \mathrm{sec}$ corresponding to the photograph shown in figure $7 \mathrm{~b}$.

Figure 10.

Volume flux $Q(L, t)$ for $h / 2 L=0.075, \omega^{2} h / g=0.136$. Numerical simulations with $N=$ 100, $\Delta t \sqrt{g / h}=0.1$. Solid line: $a / h=0.136$, dashed line: $a / h=0.1$, dotted line: $a / h=0$.

Figure 11.

Surface elevation in the shallow region above the rectangular bottom topography in natural scale for the same parameters, $h / 2 L=0.075, \omega^{2} h / g=0.136, a / h=0.136$, and time instants as for the photographs in figure 7. $N=100, \Delta t \sqrt{g / h}=0.1$. a) Time instant $t_{1}, \mathrm{~b}$ ) time instant $\left.t_{1}+0.35 \mathrm{sec}, \mathrm{c}\right)$ time instant $t_{1}+0.70 \mathrm{sec}$.

Figure 12.

Surface elevation in the shallow region above the rectangular bottom topography in natural scale for the same parameters, $h / 2 L=0.1, \omega^{2} h / g=0.22, a / h=0.15$, and time instants as for the photographs in figure 8. $N=90, \Delta t \sqrt{g / h}=0.1$. a) Time instant $t_{2}$, b) time instant $t_{2}+0.35 \mathrm{sec}$.

Figure 13.

Surface elevation for same time instants as in figures 7 and $11 . h / 2 L=0.075, \omega^{2} h / g=$ $0.136, a / h=0.1$. Solid line: Nonlinear Airy equations (without dispersion) applied in the shallow region, $N=100, \Delta t \sqrt{g / h}=0.025$. Dotted line: Boussinesq equations, $N=$ $100, \Delta t \sqrt{g / h}=0.1$. a) Time instant $t_{1}$, b) time instant $t_{1}+0.35 \mathrm{sec}$, c) time instant $t_{1}+0.70 \mathrm{sec}$.

Figure 14.

Time averaged energy flux vs. horizontal coordinate. $h / 2 L=0.075, \omega^{2} h / g=0.136, N=$ 100, $\Delta t \sqrt{g / h}=0.1$. Solid line: $a / h=0.136$, dashed line: $a / h=0.1$, dotted line: $a / h=0$. 


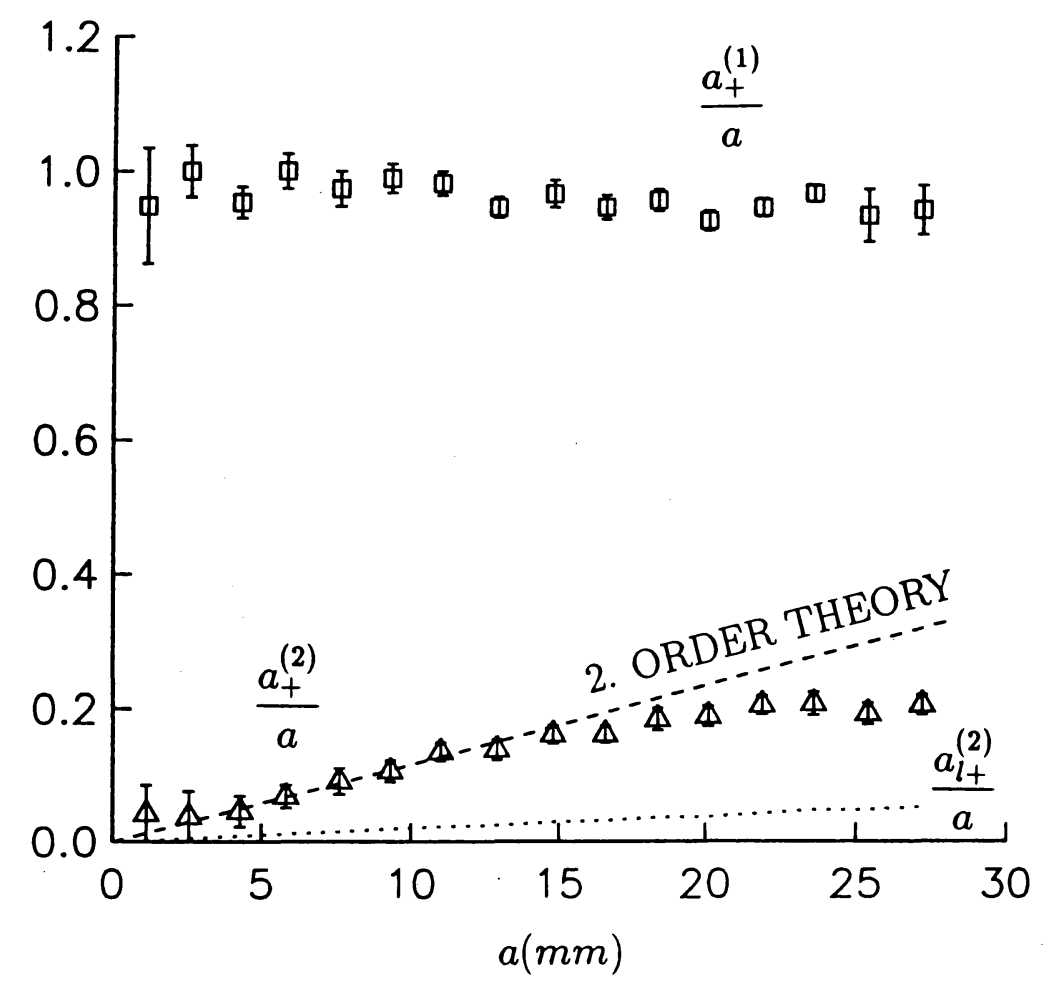

a)

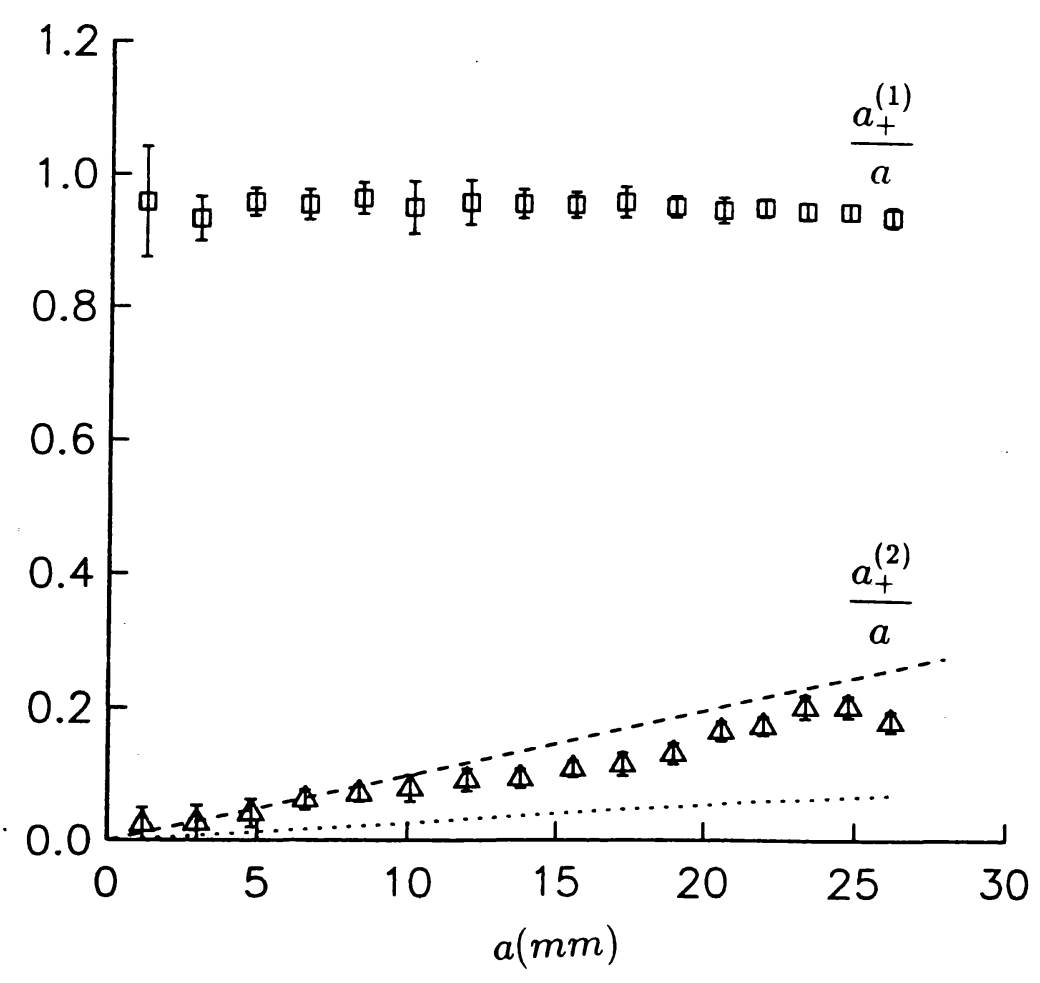

Figure 1.

Measurements of first and second harmonic free wave amplitudes at the lee side of the circular cylinder vs. incoming wave amplitude. $h=100 \mathrm{~mm}$. Squares: $a_{+}^{(1)} / a$, triangles: $a_{+}^{(2)} / a$, dotted line: $a_{l+}^{(2)} \simeq \frac{1}{2}\left(a_{+}^{(1)}\right)^{2} K / a$, dashed line: $a_{+}^{(2)} / a$ obtained by second order theory (Friis et al. 1991). a) small cylinder $(R=100 \mathrm{~mm}), \omega=2 \pi \times 1.05 \mathrm{~Hz}$. b) small cylinder $(R=100 \mathrm{~mm}), \omega=2 \pi \times 1.22 \mathrm{~Hz}$. 


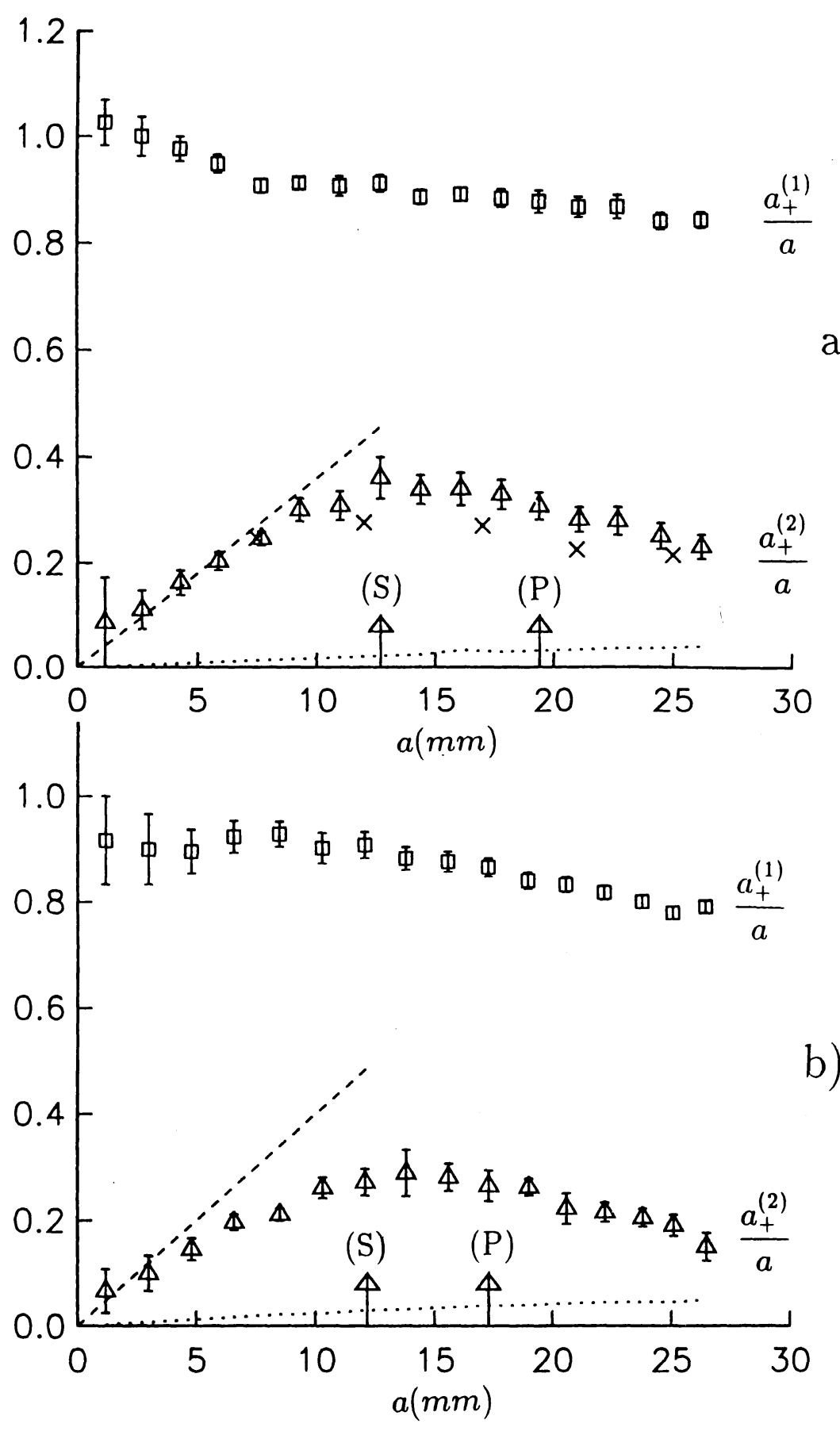

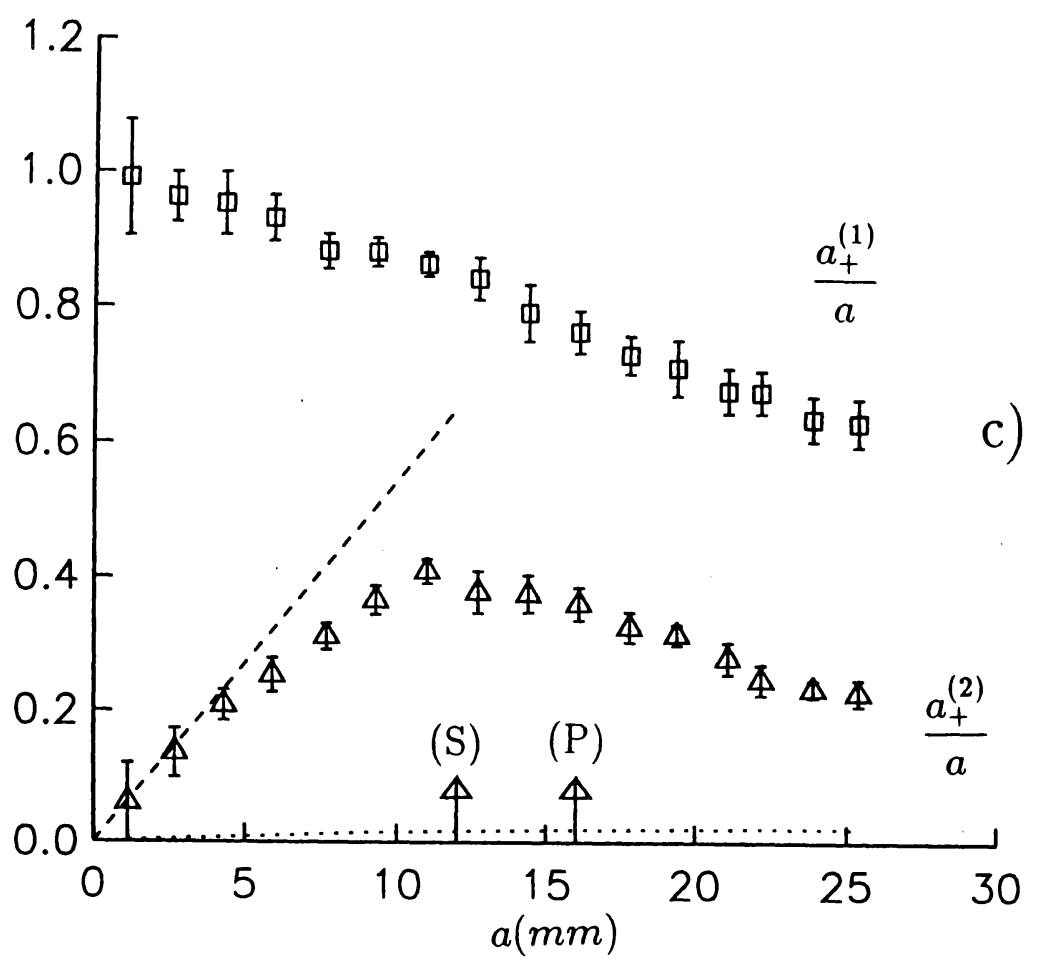

)

Figure 2.

Same as figure 1 , but $h=50 \mathrm{~mm}$. a) small cylinder $(R=100 \mathrm{~mm}), \omega=2 \pi \times 1.05 \mathrm{~Hz}$. Crosses: $a_{+}^{(2)} / a$ obtained by nonlinear theory, Cointe (1989, figure 12). b) small cylinder $(R=100 \mathrm{~mm}), \omega=2 \pi \times 1.22 \mathrm{~Hz}$. c) large cylinder $(R=190 \mathrm{~mm}), \omega=2 \pi \times 1.05 \mathrm{~Hz}$. The arrows denote respectively spilling $(S)$ and plunging $(P)$ limits. 


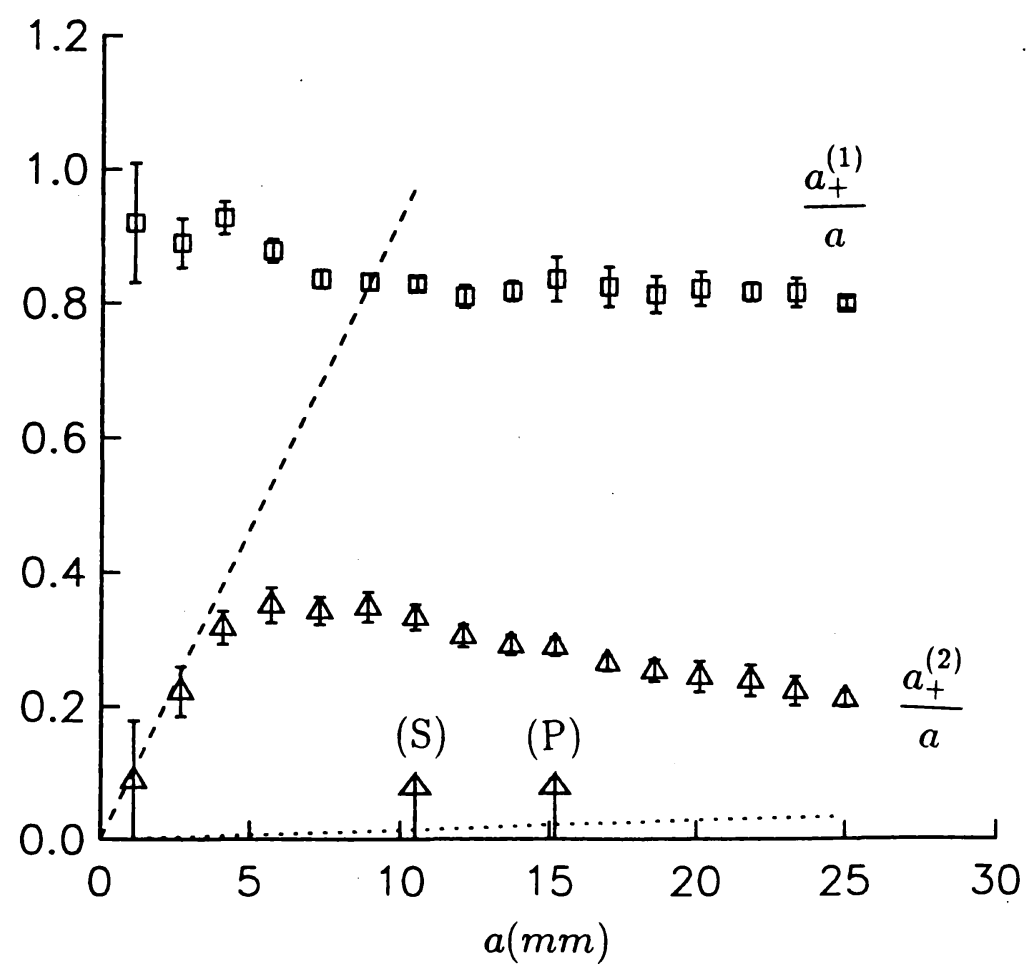

a)

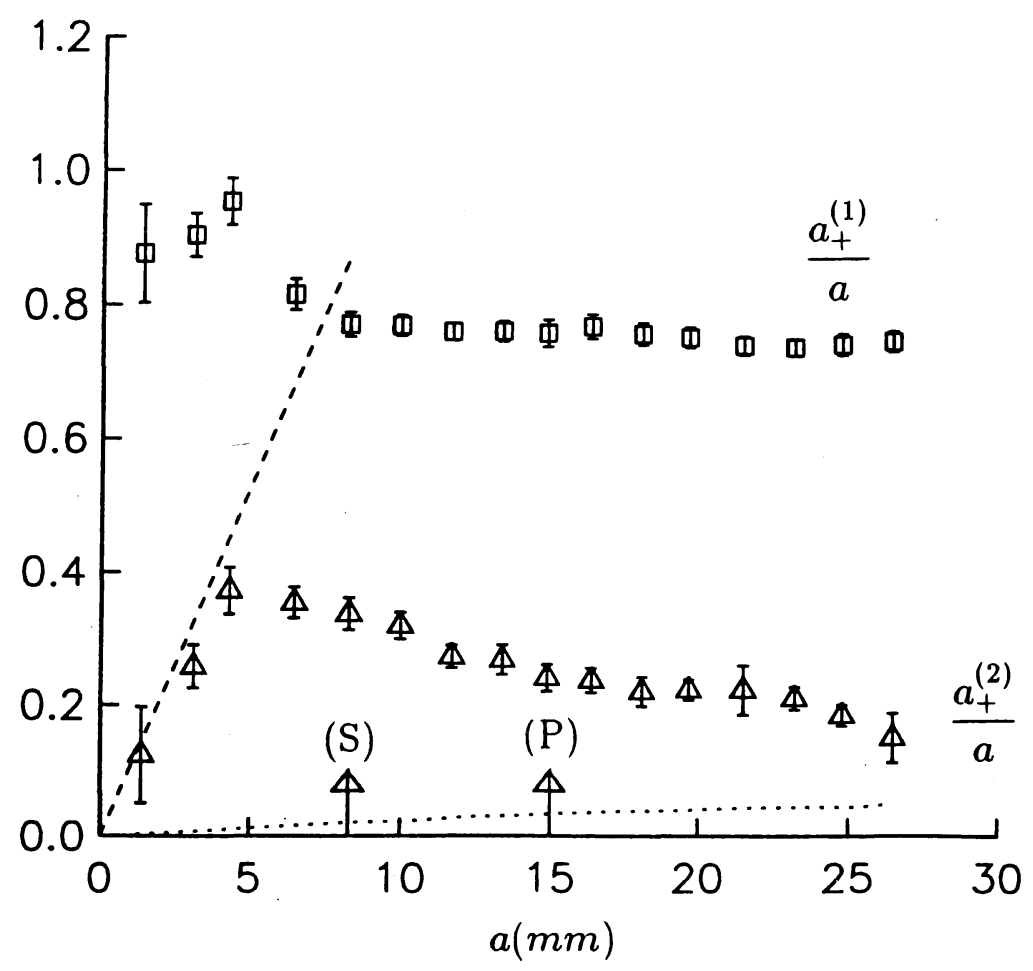

b)

Figure 3.

Same as figure 1 , but $h=25 \mathrm{~mm}$. a) small cylinder $(R=100 \mathrm{~mm}), \omega=2 \pi \times 1.05 \mathrm{~Hz}$. b) small cylinder $(R=100 \mathrm{~mm}), \omega=2 \pi \times 1.22 \mathrm{~Hz}$. The arrows denote respectively spilling $(S)$ and plunging $(P)$ limits. 


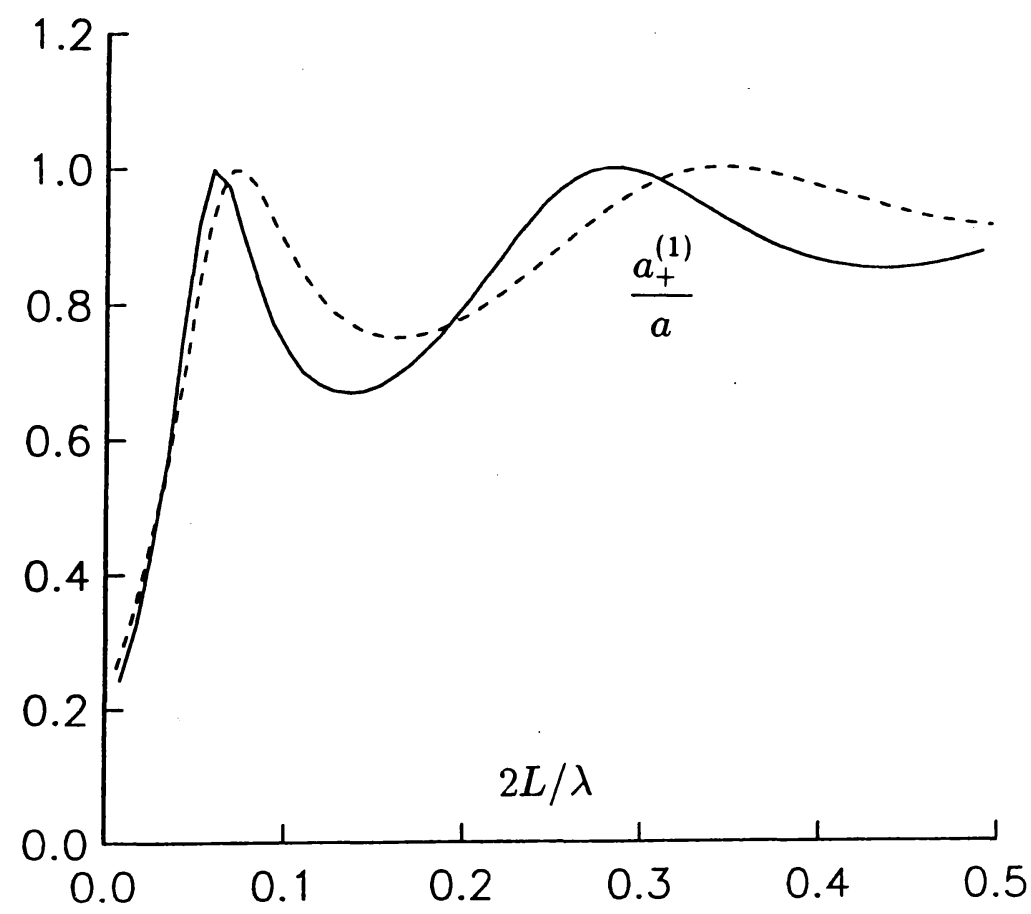

Figure 4.

Linear transmission coefficient $a_{+}^{(1)} / a$ for the rectangular bottom topography vs. $2 L / \lambda$, obtained by the theory described in $\S 4$. Solid line: $h / 2 L=0.075$, dashed line: $h / 2 L=0.1$. 


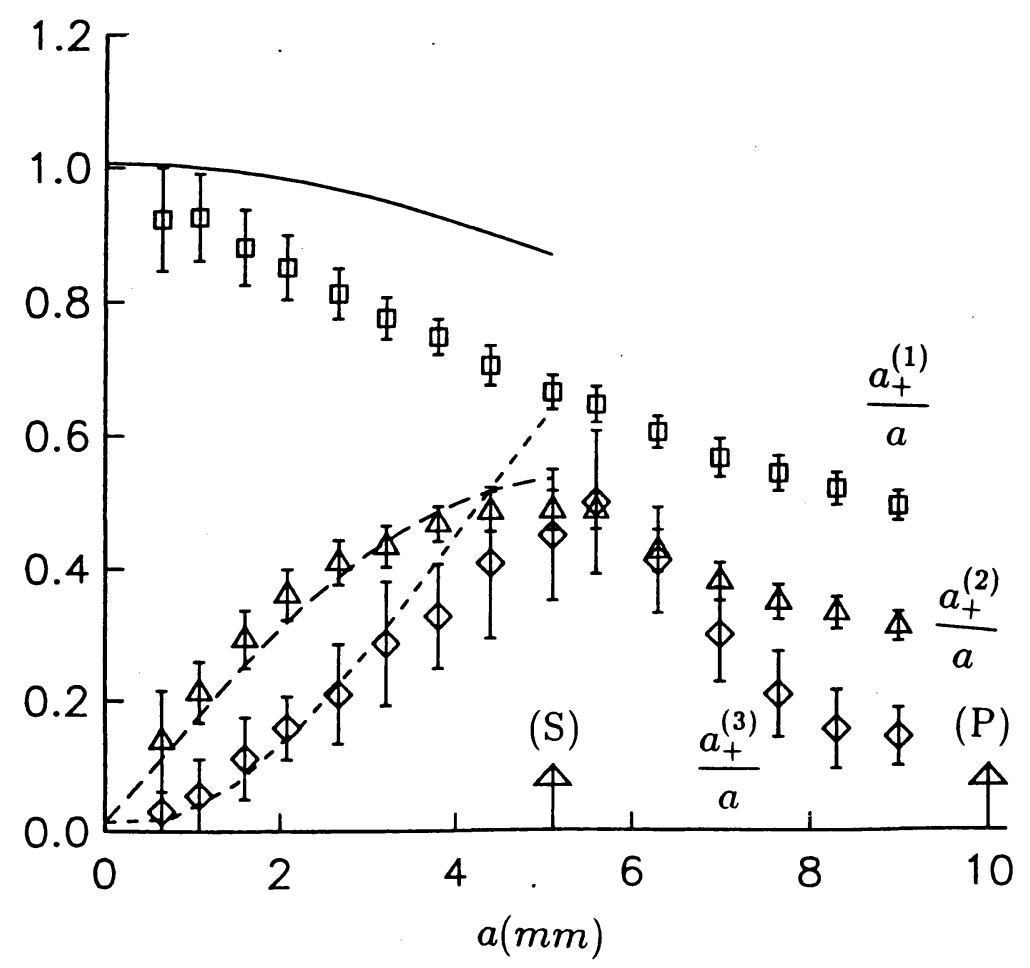

Figure 5.

First, second and third harmonic free wave amplitudes at the lee side of the rectangular bottom topography, vs. $a$, for parameter set I, i.e. $h=37.5 \mathrm{~mm}, \omega=2 \pi \times 0.95 \mathrm{~Hz}$. Measurements: Squares: $a_{+}^{(1)} / a$, triangles: $a_{+}^{(2)} / a$, diamonds: $a_{+}^{(3)} / a$. Nonlinear theory: Solid line: $a_{+}^{(1)} / a$, dashed line: $a_{+}^{(2)} / a$, dotted line: $a_{+}^{(3)} / a$. The arrows denote respectively spilling $(S)$ and plunging $(P)$ limits.

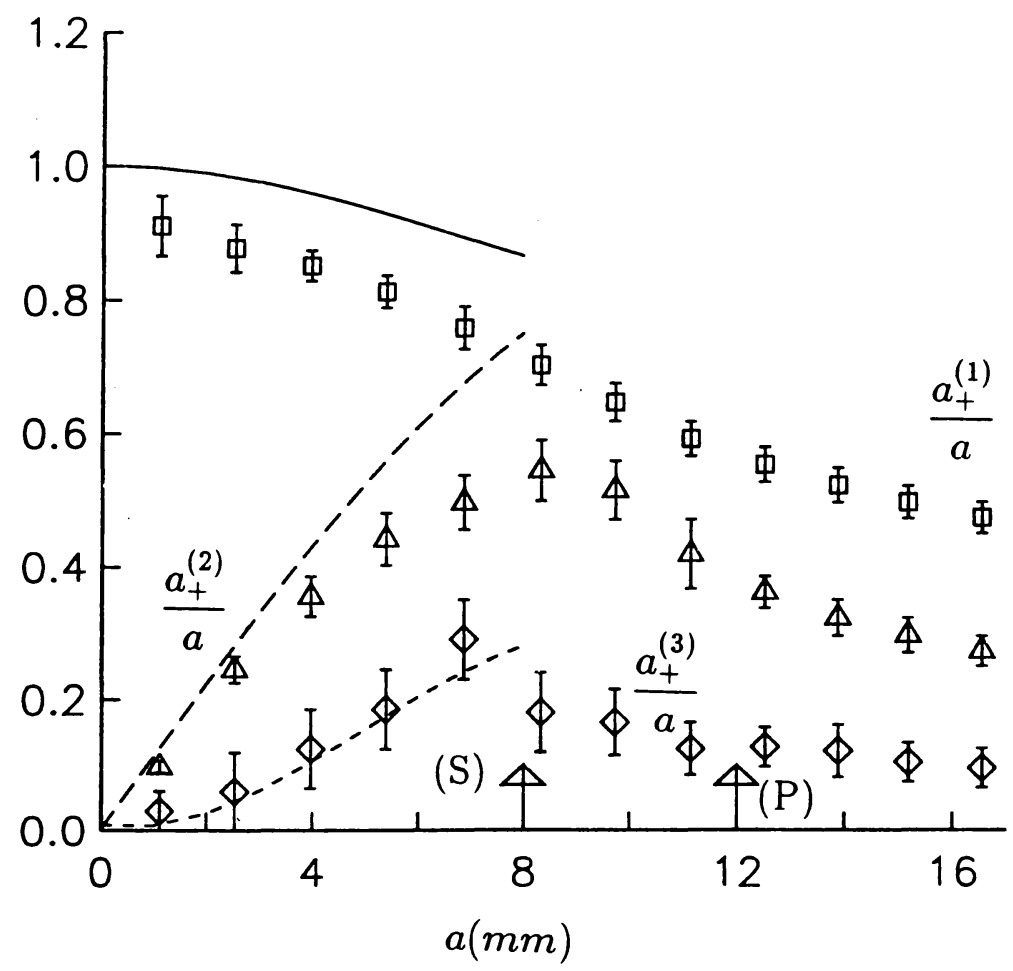

Figure 6.

Same as figure 5 , but parameter set II, i.e. $h=50 \mathrm{~mm}, \omega=2 \pi \times 1.05 \mathrm{~Hz}$. 


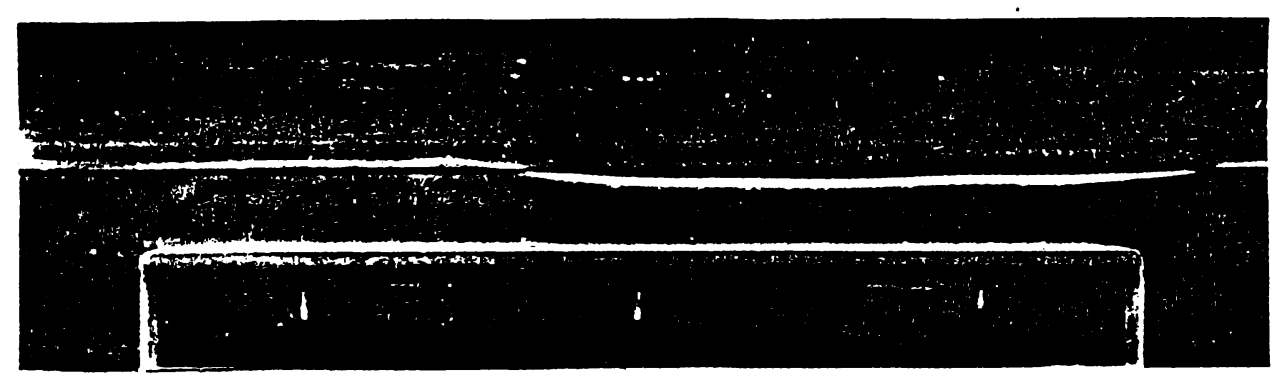

a)

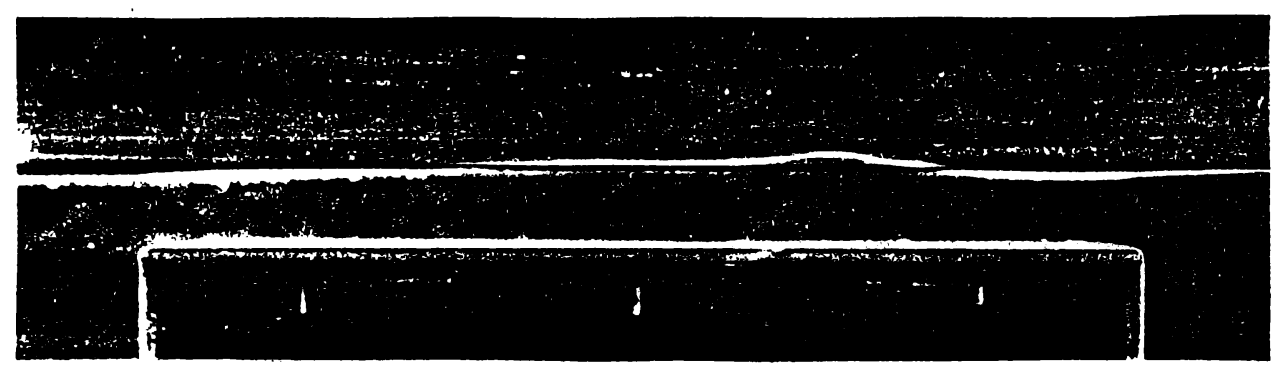

b)

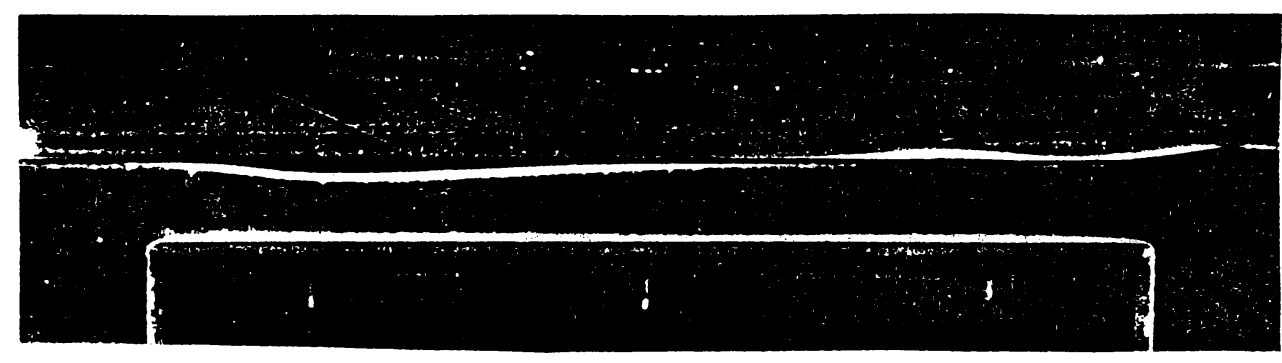

c)

Figure 7.

Photographs of the wave profile at the rectangular bottom topography for $h=37.5 \mathrm{~mm}, \omega=$ $2 \pi \times 0.95 \mathrm{~Hz}, a=5.1 \mathrm{~mm}(a / h=0.136)$. a) time instant $\left.t_{1}, \mathrm{~b}\right)$ time instant $\left.t_{1}+0.35 \mathrm{sec}, \mathrm{c}\right)$ time instant $t_{1}+0.70 \mathrm{sec}$.
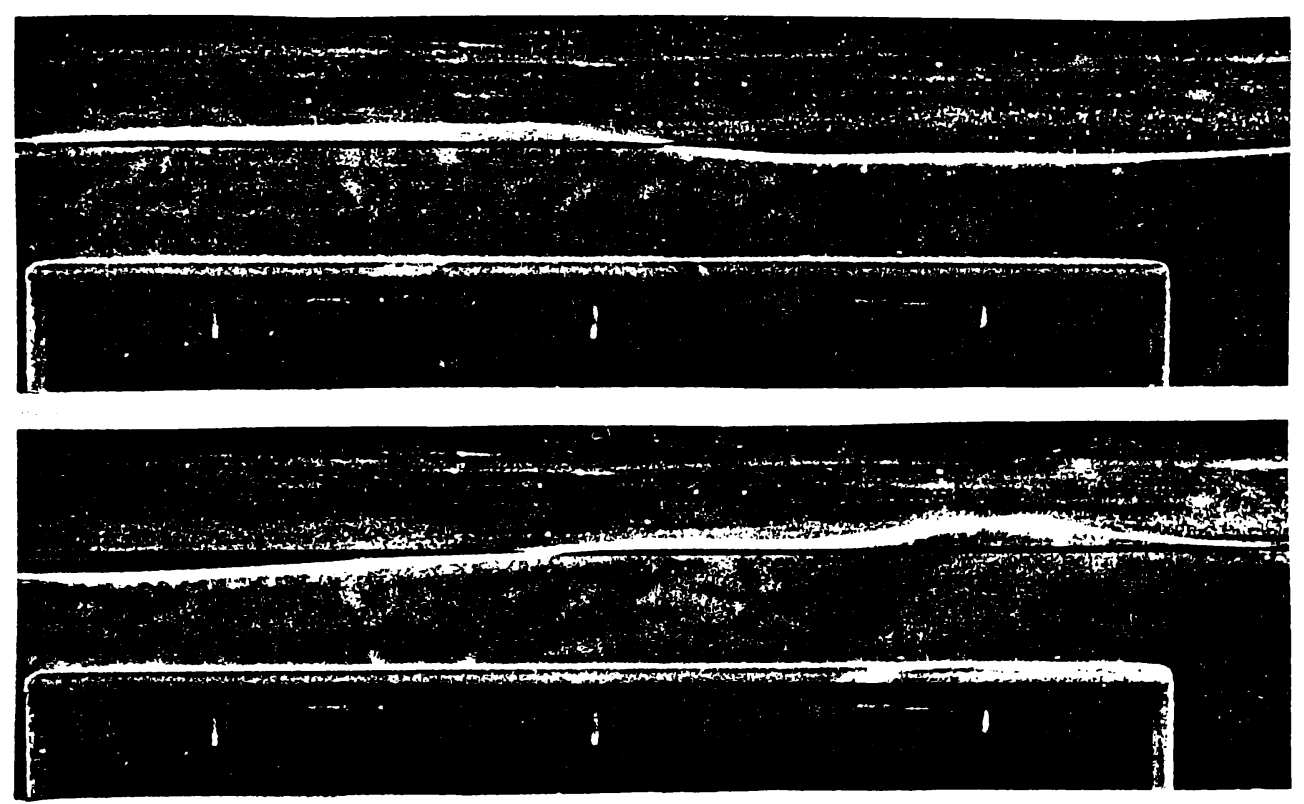

b)

Figure 8.

Photographs of the wave profile at the rectangular bottom topography for $h=50 \mathrm{~mm}, \omega=$ $2 \pi \times 1.05 \mathrm{~Hz}, a=7.5 \mathrm{~mm}(a / h=0.15)$. a) time instant $\left.t_{2}, \mathrm{~b}\right)$ time instant $t_{2}+0.35 \mathrm{sec}$. 

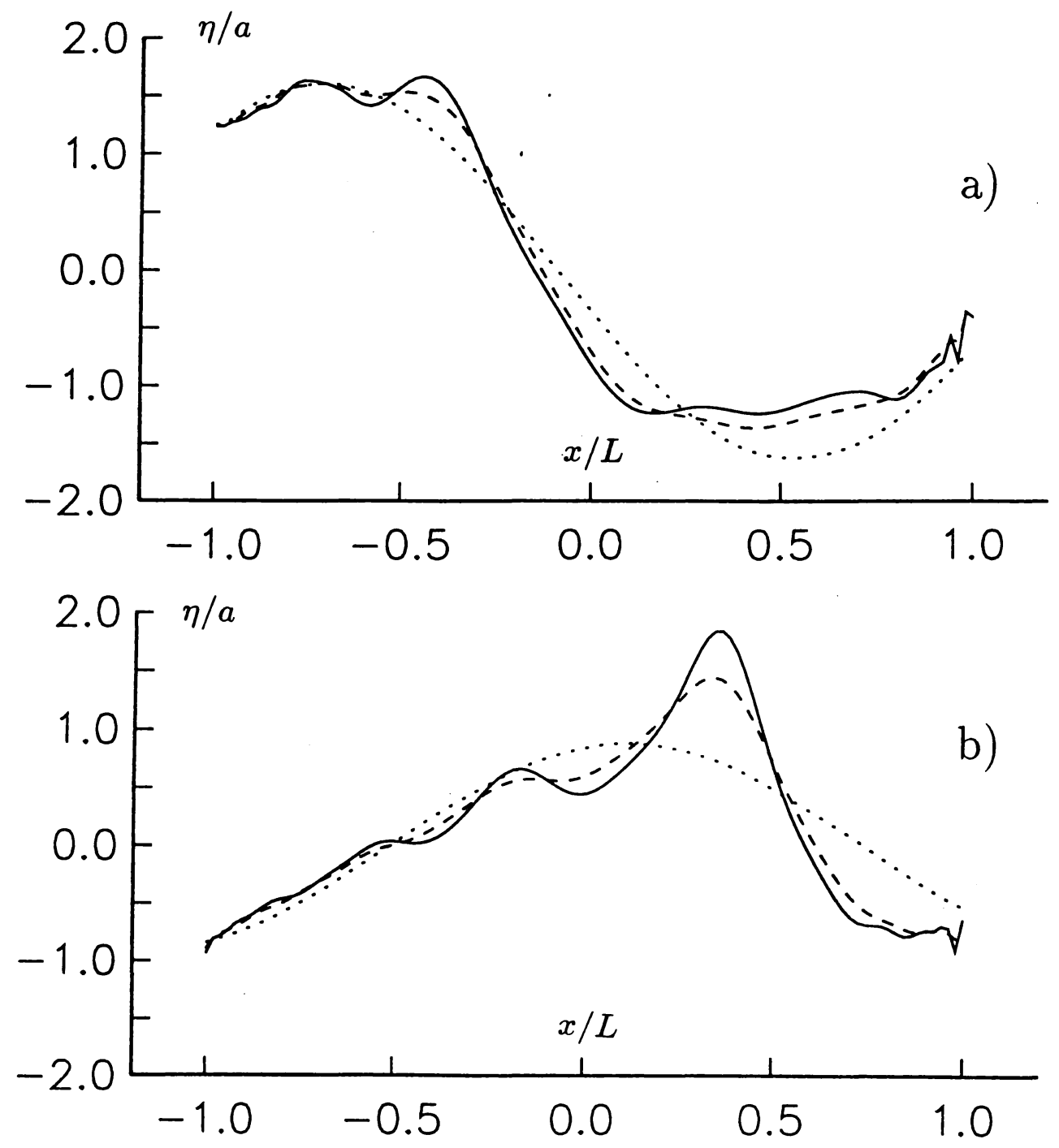

Figure 9.

Surface elevation in the shallow region above the rectangular bottom topography. $h / 2 L=$ $0.075, \omega^{2} h / g=0.136$. Numerical simulations with $N=100, \Delta t \sqrt{g / h}=0.1$. Solid line: $a / h=0.136$, dashed line: $a / h=0.1$, dotted line: $a / h=0$. a) Time instant $t_{1}$ corresponding to the photograph shown in figure $7 \mathrm{a}$. b) Time instant $t_{1}+0.35 \mathrm{sec}$ corresponding to the photograph shown in figure $7 \mathrm{~b}$.

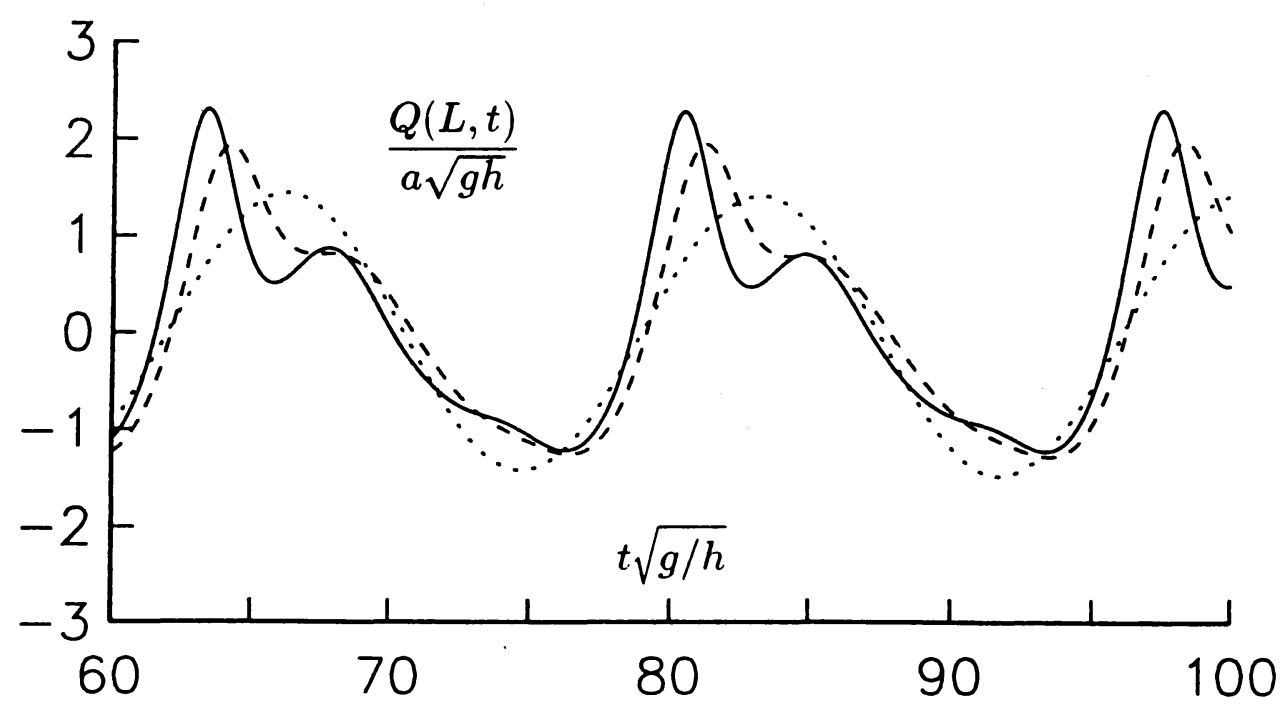

Figure 10.

Volume flux $Q(L, t)$ for $h / 2 L=0.075, \omega^{2} h / g=0.136$. Numerical simulations with $N=$ $100, \Delta t \sqrt{g / h}=0.1$. Solid line: $a / h=0.136$, dashed line: $a / h=0.1$, dotted line: $a / h=0$. 


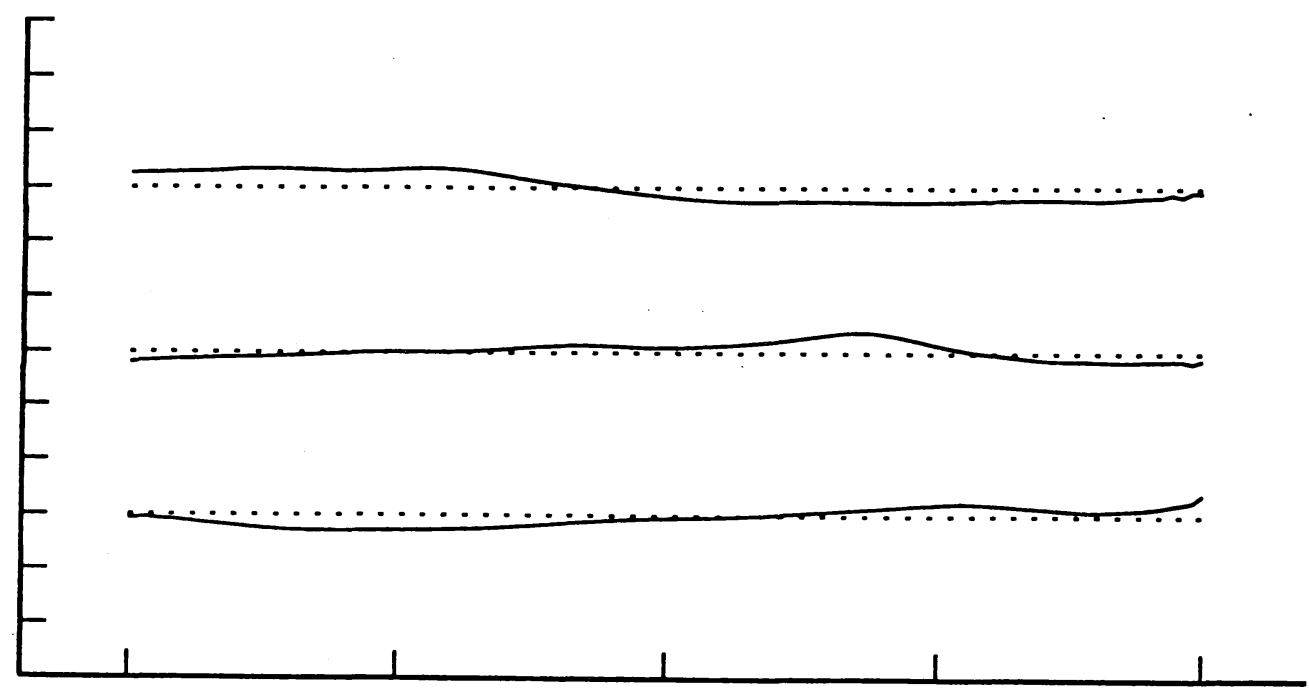

Figure 11.

Surface elevation in the shallow region above the rectangular bottom topography in natural scale for the same parameters, $h / 2 L=0.075, \omega^{2} h / g=0.136, a / h=0.136$, and time instants as for the photographs in figure 7. $N=100, \Delta t \sqrt{g / h}=0.1$. a) Time instant $\left.t_{1}, \mathrm{~b}\right)$ time instant $\left.t_{1}+0.35 \mathrm{sec}, \mathrm{c}\right)$ time instant $t_{1}+0.70 \mathrm{sec}$.

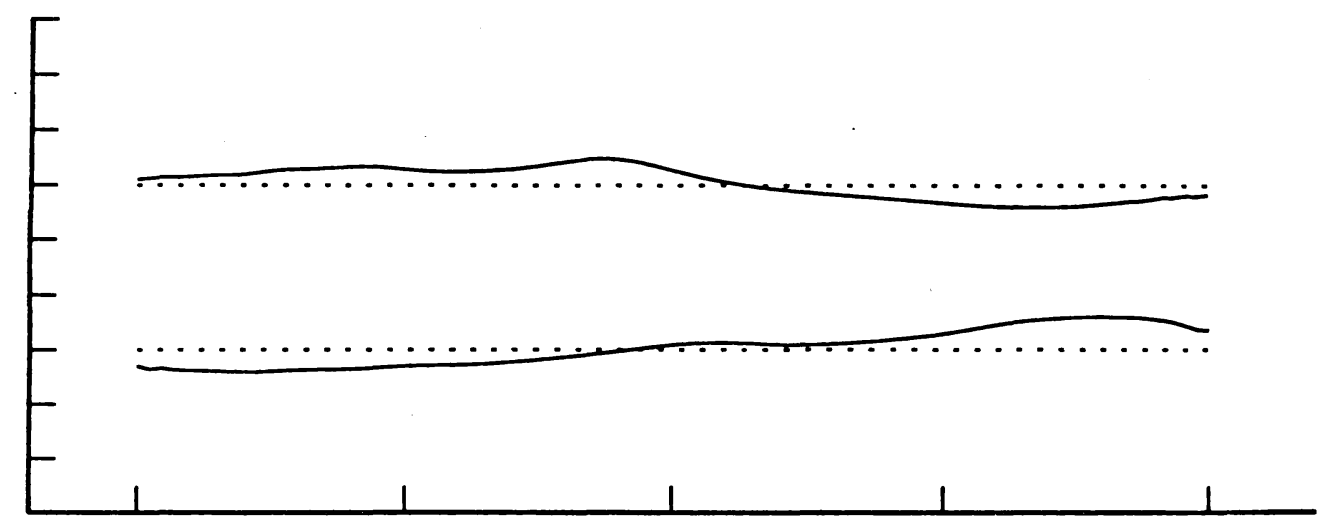

Figure 12.

Surface elevation in the shallow region above the rectangular bottom topography in natural scale for the same parameters, $h / 2 L=0.1, \omega^{2} h / g=0.22, a / h=0.15$, and time instants as for the photographs in figure 8. $N=90, \Delta t \sqrt{g / h}=0.1$. a) Time instant $t_{2}$, b) time instant $t_{2}+0.35 \mathrm{sec}$. 

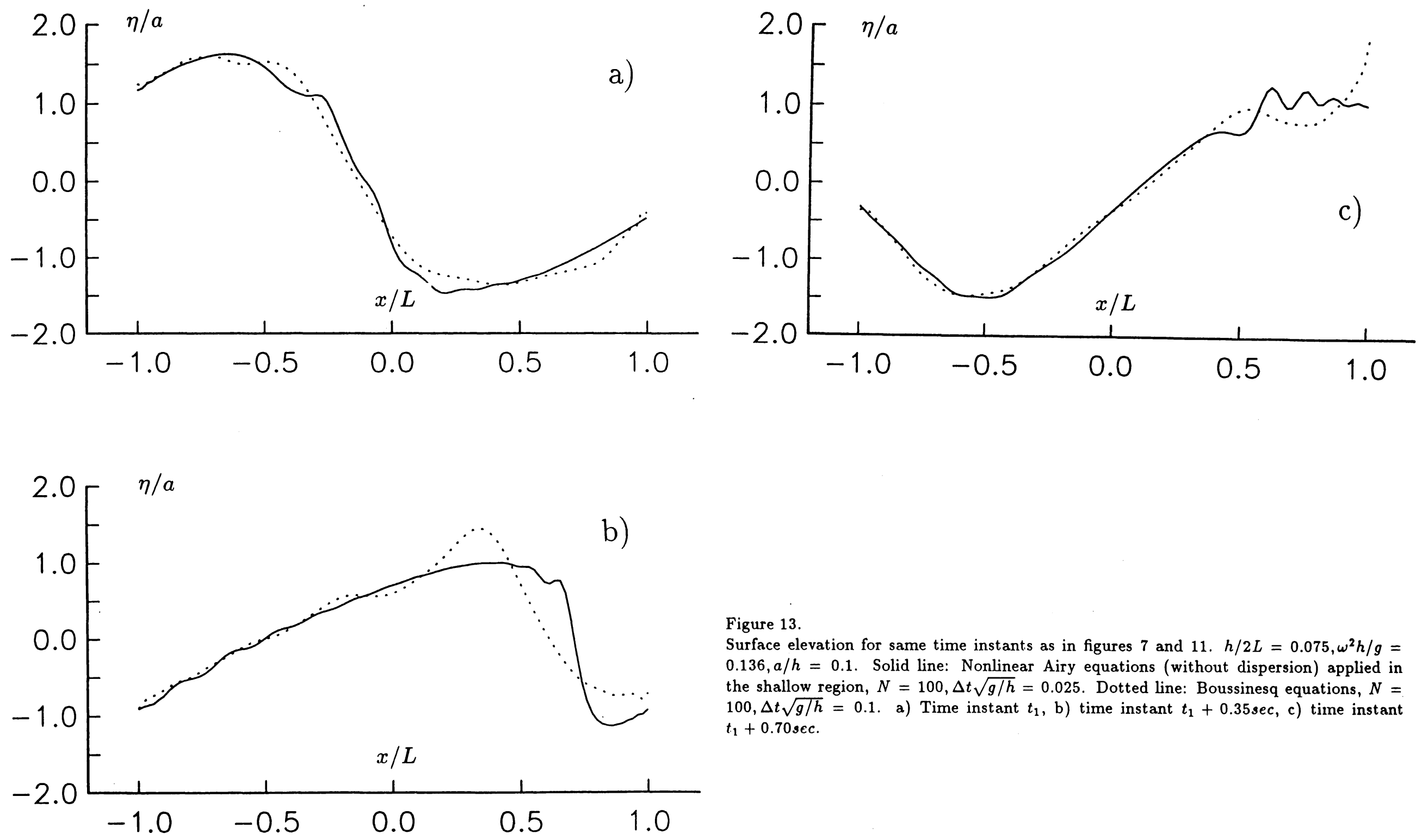

Figure 13.

Surface elevation for same time instants as in figures 7 and $11 . h / 2 L=0.075, \omega^{2} h / g=$ $0.136, a / h=0.1$. Solid line: Nonlinear Airy equations (without dispersion) applied in the shallow region, $N=100, \Delta t \sqrt{g / h}=0.025$. Dotted line: Boussinesq equations, $N=$ $100, \Delta t \sqrt{g / h}=0.1$. a) Time instant $t_{1}$, b) time instant $t_{1}+0.35 \mathrm{sec}$, c) time instant $t_{1}+0.70 s e c$. 


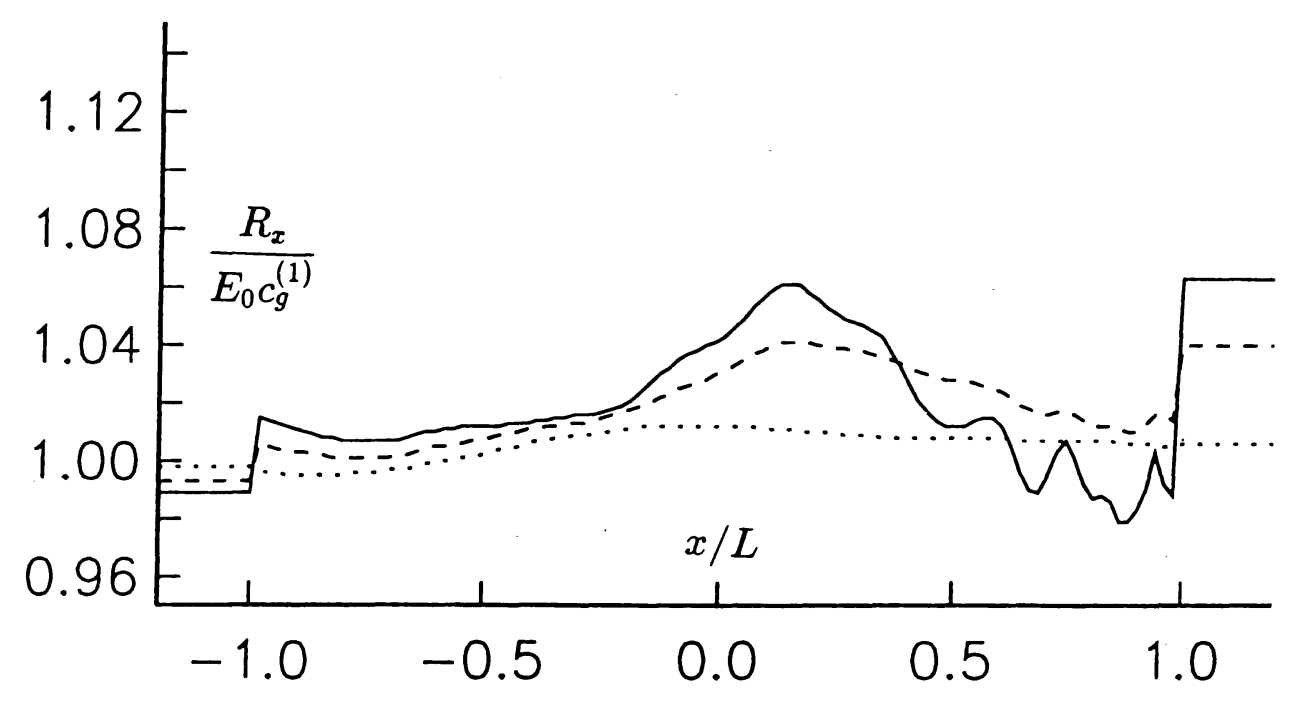

Figure 14.

Time averaged energy flux vs. horizontal coordinate. $h / 2 L=0.075, \omega^{2} h / g=0.136, N=$ $100, \Delta t \sqrt{g / h}=0.1$. Solid line: $a / h=0.136$, dashed line: $a / h=0.1$, dotted line: $a / h=0$. 
